\title{
Helping Friends or Influencing Foes: Electoral and Policy Effects of Campaign Finance Contributions*
}

\author{
Keith E. Schnakenberg ${ }^{\dagger} \quad$ Ian R. Turner ${ }^{\ddagger}$
}

February 2020

\begin{abstract}
Campaign finance contributions may influence policy by affecting elections or influencing the choices of politicians once in office. To study the trade-offs between these two paths to influence, we use a game in which contributions may affect electoral outcomes and signal policy-relevant information to politicians. In the model, a campaign donor and two politicians each possess private information correlated with a policy-relevant state of the world. The donor may allocate his budget to either an ally candidate who has relatively similar preferences or a moderate candidate whose preferences are relatively divergent from the donor's preferred policy. Contributions that increase the likelihood of the moderate being elected can signal good news about the donor's preferred policy and influence the moderate's policy choice. However, when the electoral effect of contributions is too small to demand sufficiently high costs to deter imitation by groups with negative information, this informational effect breaks down.
\end{abstract}

${ }^{*}$ The authors would like to thank Michael Nelson, John Patty, Cathy Hafer, Sandy Gordon, Stephane Wolton, Carlo Prato Chris Cotton, Arnauld Dellis, Tom Groll and seminar participants at New York University, the Harris School of Public Policy, Columbia University, Texas A\&M University, University of Kentucky Department of Economics, University of Rochester, California Institute of Technology, Stanford Graduate School of Business, Queens University, and participants in the CSAP conference at Yale University. All errors are ours.

${ }^{\dagger}$ Assistant Professor of Political Science, Washington University in St. Louis, keschnak@wustl.edu.

$¥$ Assistant Professor of Political Science, Yale University, ian.turner@yale.edu. 
Running for office in the United States is expensive. The 2016 United States Presidential and Congressional elections cost just under 6.5 billion dollars - over one-fifth of the GDP of Vermont - with the inflation-adjusted cost of Congressional elections increasing by around $45 \%$ since 2008 and $73 \%$ since $2000 .^{1}$ Furthermore, the rise of unlimited soft money has allowed a larger proportion of campaign funds to be provided by a smaller set of elite donors and groups. What do donors think they are getting in return for spending so much money? A good answer to this question would further inform our understanding of the role of interest groups in modern policymaking, shape our views about whether or not the current campaign finance regime is harmful to representation, and allow us to formulate better predictions about the effects of reform proposals.

Extant literature provides several possible answers to the question of donor motivations, though none are satisfying on their own. The key motivations of large donors fall into two categories: influencing electoral outcomes and influencing the behavior of politicians. A pure electoral model assumes that donors take politicians' preferences and behavior as given and spend money to help the electoral prospects of like-minded politicians (e.g., Poole, Romer and Rosenthal 1987). Alternatively, contributions may influence the behavior of politicians either through quid pro quo exchange (e.g., Grossman and Helpman 1994) or by signaling policy-relevant information to policymakers (e.g., Potters and van Winden 1992).

Each of these accounts provides an incomplete picture of the influence of contributions. For instance, the pure electoral and quid pro quo models imply that donors should strictly benefit when the candidate they backed wins office, a prediction for which there is no evidence at least corporate contributions (Fowler, Garro and Spenkuch 2019). Furthermore, there is little evidence of a connection between contributions and the behavior of individual legislators, suggesting limited empirical support for a quid pro quo model (Ansolabehere, de Figueiredo and Snyder 2003). Signaling models provide a better account of these patterns, since a signal of policy information affects politicians who did not receive the contribution, but some questions remain unanswered:

\footnotetext{
${ }^{1}$ The inflation-adjusted dollar amount for 2016 federal election spending is $\$ 6,444,253,265$, which is approximately a $50 \%$ increase in overall spending in federal elections compared to 2000 (Data retrieved from Open Secrets on June 7, 2018 at https: / / www. opensecrets.org/overview/cost.php?display=T\&infl=Y).
} 
What makes campaign contributions useful informational devices as opposed to some other conspicuous expenditure (e.g. charitable contributions, sponsorships, advertising)? How can we explain the targeting of informational contributions? Our answers to these questions are based on the idea that influencing elections and influencing politicians are interrelated goals. If a donor's policy-relevant information affects their perceived stake in the election then the electoral effects of contributions may contribute to the costs that allow contributions to serve as credible signals. This makes campaign contributions uniquely useful signals and also pushes some donors to contribute to the candidate they least prefer on electoral grounds in order to signal favorable information.

To illustrate our argument, consider the example of a political action committee (PAC) for a trade association that seeks to avoid expensive regulation of a product produced by its members. A continuum of regulatory policies may be proposed and implemented, ranging from no regulations to an outright ban on the product. A coming election will determine who will take office and make this key decision, and the race is between two candidates who we will label the Ally and the Moderate. The Ally, like the trade association, prefers zero regulation. The Moderate's policy preferences depend on the potential dangers of the product, though this information is unknown to the players. Through internal studies, the trade association learns noisy private information about the dangers of the product. If this information suggests the product is dangerous we will say the PAC is a bad type and otherwise we will say the PAC is a good type. Furthermore, the winner of the elections will independently receive noisy information about the safety of the product.

The PAC decides whether to contribute to the Ally candidate or the Moderate candidate. Contributions have a positive effect on the probability the recipient wins the election. Thus, from an electoral perspective, the PAC clearly prefers to contribute to the Ally's campaign. However, contributions are also publicly observable and may convey the PAC's private information about its client's product. This consideration will sometimes cause the PAC to contribute to the Moderate in order to convince her that it has favorable information.

For contributions to be a credible signal of information the good type of PAC must be more willing than the bad type to contribute to the Moderate in order to convince her its signal was 
good. This is true in this example for two reasons. First, the PAC's information helps it predict what signal the Moderate will receive if she gains office. If the PAC receives a bad signal then it believes the Moderate's signal will likely also be bad. The bad type of PAC therefore expects to be more heavily regulated by the Moderate than the good type even if it manages to successfully misrepresent its own information. Second, the expected difference between the Moderate's and Ally's policy is larger when the product is expected to be more dangerous. This means that the bad type places relatively higher weight on electoral rather than signaling aspects of influence and therefore will not trade away electoral influence for behavioral influence as readily as the good type. Thus, the trade-off between influencing elections and influencing politicians' behavior induces payoffs to the PAC that allow for credible information transmission through contributions.

To generalize this logic we analyze a model of large campaign contributions in which donors are motivated by influencing electoral outcomes as well as influencing the behavior of politicians. We provide a general argument for why the electoral effects of campaign contributions make them uniquely useful signals of policy information and create opportunities for information transmission that may not otherwise exist. The key mechanism is the one described in the example above: a donor who has a good case on policy grounds expects opposing politicians to be more persuaded to move policy in their direction and is therefore more willing to forgo opportunities to put allies in positions of power. These donors therefore distinguish themselves to politicians by forgoing opportunities to provide electoral benefits to their allies. Thus, we provide an empirically plausible account of informative campaign contributions that explains why campaign expenditures may be particularly useful compared to other expenditures. Furthermore, the theory offers predictions about the distribution of these expenditures across candidates and races, as well as insight into several important empirical questions in the literature.

\section{Existing accounts of campaign contributions}

We explain donor behavior as a mixture of electoral motivation and signaling to policymakers. These two effects have been analyzed in isolation but, as we will demonstrate, the combination of 
electoral and signaling effects leads to unexpected insights that help explain empirical patterns in donor behavior.

Electoral effects. A first-order effect of campaign contributions is that money helps win elections. Empirical work on aggregate campaign spending (Erikson and Palfrey 2000; Hall 2016) as well as experimental analyses of specific uses of campaign dollars (see Green and Gerber 2015) show that campaign spending is effective. The demonstrable effects of campaign spending on electoral outcomes provide an opportunity for donors to influence policy by boosting the election prospects of like-minded politicians. Empirical analysis of contribution behavior provides indirect evidence that donors consider electoral effects when allocating contributions. For instance, Bonica (2013) and Barber, Canes-Wrone and Thrower (2017) show that individual donors allocate more contributions to ideologically close politicians in close races. Furthermore, surveys of individual donors show that they consistently rate "to affect election outcome" as a top reason for donating (Barber 2016). Models of pure electoral motivation also cannot explain why firms appear not to benefit from backing the winner of an election though, as Fowler, Garro and Spenkuch (2019) note, this finding is consistent with many signaling models of contributions, including the one in this paper. Overall, the evidence suggests the need for a theory that includes some electoral motivation but allows other motivations to operate simultaneously.

Other research also studies the impact of special interest influence on both electoral outcomes and policy (Felli and Merlo 2007; Sasso and Alexander 2019). ${ }^{2}$ For example, Felli and Merlo (2007) highlights the trade-off between electing friends and lobbying enemies. They study a citizen-candidate model in which interest groups may donate campaign funds and make ex post transfers in exchange for policy favors. In equilibrium, groups only donate campaign funds to their most preferred candidate and only make ex post transfers when their least preferred candidate wins the election. Thus, interest groups either influence electoral outcomes or buy policy, but not both.

\footnotetext{
${ }^{2}$ In Sasso and Alexander (2019) groups can give contributions before an election and lobby the winner after the election. The dollar amounts of contributions are influenced in part by expectations about payoffs from the lobbying stage.
} 
Signaling to policymakers. Another important way that campaign contributions are theorized to influence policymakers is by transmitting information. ${ }^{3}$ This idea appears in a handful of papers which differ with respect to the nature of the information being transmitted and the actions taken by the policymaker. In some informational models contributions pay for access to the politician and additional information may be transmitted once access is granted (Austen-Smith 1995, 1998; Cotton 2012, 2016; Lohmann 1995; Potters and van Winden 1992). Of these, our paper relates most closely to Cotton $(2009,2016)$ in that the politician's access to information limits groups' temptation to exaggerate. In other models, as in this one, contributions serve as signals aimed at influencing policy choices directly, such as in Gordon and Hafer (2005) and Gordon and Hafer $(2007) .^{4}$

In the models discussed above, the important characteristics of campaign contributions are that they are public and costly. Because many other activities have these characteristics, existing models do not necessarily uniquely predict that campaign contributions serve as signals. For instance, Gordon and Hafer (2005) note that other conspicuous expenditures (e.g., donating to charities) would be consistent with the theoretical mechanism highlighted in that paper, and remain agnostic as to whether the political expenditures they describe are lobbying expenditures or campaign contributions. Similarly, in other papers campaign spending is simply costly actions taken (Austen-Smith 1995) or transfers to politicians (Cotton 2012). Our model differs from the literature in this respect. The signaling value of contributions comes not from their direct costs but from their electoral effects. Thus, our model better explains why, under some circumstances, campaign contributions are uniquely good signaling devices relative to other expenditures.

\footnotetext{
${ }^{3}$ Here we focus on campaign contributions as mechanisms for transmitting information to politicians. The literature also considers that campaign contributions play a role in transmitting information to voters (Ashworth 2006; Coate 2004; Prato and Wolton 2017).

${ }^{4}$ Other models with multiple channels of interest group influence include Fox and Rothenberg (2011), in which electorally-motivated contributions may indirectly influence policy, and Wolton (2018) in which inside lobbying changes policy by signaling ability to engage in outside lobbying.
} 


\section{A model of campaign contributions}

Players, types, and sequence of play. We model a situation in which a single campaign donor may spend money to influence the outcome of a two-candidate election. The players are a Moderate candidate $M$, an Ally candidate $A$, and a campaign Donor $D$. The 'Donor' can be conceptualized as an interest group such as certain unions, corporate PACs, trade associations, social welfare organizations, or an influential mega-donor with a known agenda. We index players with $i \in$ $\{M, A, D\}$ and use $-i$ to denote the set of all players other than $i$.

The set of feasible policies is $X=[0,1]$, where smaller numbers denote policies that are more favorable to the Donor. Thus, we may interpret $x \in X$ to be the intensity of regulation of the industry represented by the Donor, assuming that the industry prefers to avoid regulation. Additionally, there is a state of the world $\theta \in[0,1]$ that may affect players' policy preferences. The common prior belief is that $\theta$ is distributed according to a continuous distribution with density $f$ and full support on $[0,1]$. If the policy choice is interpreted as the intensity of regulation, then $\theta$ can be interpreted as some piece of information that would affect the public's demand for regulation, such as information about a product's safety or its potential environmental impacts.

The sequence of the game is as follows. First, all players receive independent, noisy signals $s_{i} \in\{G, B\}$ about the state of the world, where $\operatorname{Pr}\left[s_{i}=B \mid \theta\right]=\theta$. A signal $s_{i}=G$ denotes a "good" signal from the Donor's perspective and $s_{i}=B$ denotes a "bad" signal. That is, $s_{i}=G\left(s_{i}=B\right)$ suggests that lower (higher) policy is more likely to be optimal, which is preferred (opposed) by the Donor. The signals are private information. Second, the Donor chooses contribution levels $c=\left(c^{M}, c^{A}\right)$ where $c^{M}$ is the contribution to the Moderate and $c^{A}$ is the contribution to the Ally. We assume that $c^{M}+c^{A} \leq 1$, which implies the Donor has a political budget of one that he can allocate across the Moderate and the Ally. The probability of the Moderate candidate winning the election is a continuous function $p\left(c^{M}, c^{A}\right)$ that is increasing in $c^{M}$, and decreasing in $c^{A}$. The

probability of the Ally winning is then $1-p\left(c^{M}, c^{A}\right)$. Finally, the winner of the election chooses a policy $x \in X$. 
Players' preferences. The Donor's preferences are independent of the state of the world: he prefers less regulation to more regulation for any $\theta$. His preferences are represented by the utility function,

$$
u_{D}(x)=-x
$$

The candidates are policy motivated and their preferences are assumed to satisfy three properties. First, the candidates prefer more regulation when they believe $\theta$ is larger. Second, the Ally's preferences are more aligned with the Donor's than are the Moderate's preferences. Third, the Ally is less sensitive to information about $\theta$ than the Moderate. The three properties described above are represented in a stylized manner with the following utility functions:

$$
\begin{aligned}
& u_{M}(x, \theta)=-(x-\theta)^{2}, \\
& u_{A}(x, \theta)=-(b x-\theta)^{2},
\end{aligned}
$$

where $b>1$ represents the difference in how the candidates wish to relate policy to the state of the world. One intuitive property of the candidates' policy preferences is that they are relatively similar when $\theta$ is believed to be small and diverge as $\theta$ increases. For instance, if both candidates believe that a particular pesticide poses no threat to public safety $(\theta=0)$, then they would both agree to zero regulation. Only if they learned of potential harm from the pesticide would candidate preferences diverge, perhaps because they would trade off economic and public-safety benefits at different rates.

Equilibrium concept. The analysis focuses on pure strategy perfect Bayesian equilibria (PBE). A pure strategy for the Donor is a function $\sigma_{D}:\{G, B\} \rightarrow[0,1]^{2}$ that maps signals into contribution decisions. A pure strategy for a candidate $i \in\{M, A\}$ is a function $\sigma_{i}:\{G, B\} \times[0,1]^{2} \rightarrow X$ that maps the candidate's signal and the Donor's contribution decisions into policy choices. Beliefs over $\theta$ and $s_{-i}$ are denoted by joint distribution functions $\mu\left(\theta, s_{-i} \mid \cdot\right)$ which are players' conditional beliefs about the state $\theta$ and the signals of other players given their own signals and, in the case of candidates, observed contributions by the Donor. Given beliefs $\mu\left(\theta, s_{-i} \mid \cdot\right)$, we denote the marginal 
belief about $\theta$ as $F(\theta \mid \cdot)=\sum_{s_{-i} \in\{G, B\}^{2}} \mu\left(\theta, s_{-i} \mid \cdot\right)$ and its density as $f(\theta \mid \cdot)$. A PBE is a strategy profile and system of beliefs such that players' strategies are sequentially rational and beliefs are consistent with Bayes' rule on the path of play.

\section{Comments on modeling assumptions}

Though our intention is to provide a model of campaign contributions, the model is flexible enough to describe other forms of electorally useful activities. For instance, endorsements from specific groups may have meaningful effects in some elections (Arceneaux and Kolodny 2009) and the donor may vary the intensity of these endorsements to induce different effects. Additionally, Hertel-Fernandez (2018) documents how corporations increasingly mobilize their own employees into politics. None of the results in this paper rely on the fact that campaign contributions are financially costly: the factors leading to information revelation by donors arise endogenously through electoral effects rather than costs of contributions, so the costless contributions in our model still reveal information. In the "Costly contributions" section below we include direct costs of contributions and show that the main results remain unchanged.

For convenience, we assume that the signals of the players are independent conditional on the state of the world. However, the main results are driven by the fact that signals are positively correlated from the players' perspectives since they cannot condition on the state of the world. If we relaxed the conditional independence assumption, positive conditional correlation of signals would tend to work in favor of our main results and negative conditional correlations would tend to work in the opposite direction.

We do not include a strategic voter in the model and simply assume that a contribution to a candidate increases the probability that candidate wins the election. We do not think of the electoral effects of contributions as being generated by voters using them to update their beliefs about $\theta$. There are typically many issues at stake in a campaign and many donors such as interest groups specialize in policy areas that are relatively low salience to the voter. Furthermore, the voter may not be aware of the source of contributions. One way to think about campaign contributions 
is as a factor that contributes valence (i.e., non-policy appeal) to the candidate (Meirowitz 2008).

\section{Equilibrium analysis}

\section{Posterior beliefs}

A preliminary step to characterizing equilibria is to derive players' beliefs at each information set given the strategies of the other players.

Donor beliefs. The Donor observes his own signal $s_{D}$ but no actions of other players. Thus, the Donor forms beliefs about the state of the world using his signal and beliefs about other players' signals follow from his beliefs about the state. Specifically, the Donor updates beliefs about $\theta$ given $s_{D}$ using Bayes' rule. The conditional density is,

$$
f\left(\theta \mid s_{D}\right)=\frac{f(\theta) \theta^{\mathbb{I}_{\left(s_{D}=B\right)}}(1-\theta)^{1-\mathbb{I}_{\left(s_{D}=B\right)}}}{\int_{0}^{1} f(\tilde{\theta}) \tilde{\theta}^{\mathbb{I}_{\left(s_{D}=B\right)}}(1-\tilde{\theta})^{1-\mathbb{I}_{\left(s_{D}=B\right)}} d \tilde{\theta}} .
$$

The conditional expectation of $\theta$ is always higher given a signal of $B$ than given a signal of $G$. Given these beliefs about $\theta$, the probability that some politician $j$ receives a signal of $s_{j}=B$ conditional on the Donor receiving signal $s_{D}$ is,

$$
\operatorname{Pr}\left[s_{j}=B \mid s_{D}\right]=\int_{0}^{1} \theta f\left(\theta \mid s_{D}\right) d \theta=\mathbb{E}\left[\theta \mid s_{D}\right] .
$$

The probability that the Donor assigns to candidates receiving a bad signal, which is unfavorable given his preference for lower regulation, is simply equal to the Donor's posterior expectation of $\theta$ given $s_{D}$. Thus, bad types of Donors believe politicians are also more likely to get $B$ signals.

Candidate beliefs. The candidate who takes office observes her own signal as well as the Donor's contributions. Both pieces of information may inform her beliefs about $\theta$. Since we are focused on pure strategy equilibria there are only two possibilities. The first is that contributions reveal nothing about the Donor's signal, in which case a candidate updates only on her own signal as in Equation (4) (substituting $s_{A}$ or $s_{M}$ for $s_{D}$ ). The second possibility is that contributions fully 


\begin{tabular}{lccc}
\hline Type of equilibrium & $\begin{array}{c}\text { Information sets: } \\
\hat{s}_{D} \text { and } s_{j}\end{array}$ & $\begin{array}{c}\text { Conditional densities: } \\
f(\theta \mid \cdot)\end{array}$ & $\begin{array}{c}\text { Conditional expectations: } \\
\mathbb{E}[\boldsymbol{\theta} \mid \cdot]\end{array}$ \\
\hline Pooling equilibria & $s_{j}=G$ & $\frac{f(\theta)(1-\theta)}{\int_{0}^{1} f(\hat{\theta})(1-\hat{\theta}) d \hat{\theta}}$ & $\frac{\mathbb{E}[\theta]-\mathbb{E}\left[\theta^{2}\right]}{1-\mathbb{E}[\theta]}$ \\
\hline \multirow{2}{*}{ Separating equilibria } & $s_{j}=B$ & $\frac{f(\theta) \theta}{\int_{0}^{1} f(\hat{\theta}) \hat{\theta} d \hat{\theta}}$ & $\frac{\mathbb{E}\left[\theta^{2}\right]}{\mathbb{E}[\theta]}$ \\
& $\hat{s}_{D} \neq s_{j}$ & $\frac{f(\theta)(1-\theta) \theta}{\int_{0}^{1} f(\hat{\theta}(1-\hat{\theta}) \hat{\theta} d \hat{\theta}}$ & $\frac{\mathbb{E}\left[\theta^{2}\right]-\mathbb{E}\left[\theta^{3}\right]}{\mathbb{E}[\theta]-\mathbb{E}\left[\theta^{2}\right]}$ \\
& $\hat{s}_{D}=s_{j}=G$ & $\frac{f(\theta)(1-\theta)^{2}}{\int_{0}^{1} f\left(\hat{\theta}(1-\hat{\theta})^{2} d \hat{\theta}\right.}$ & $\frac{\mathbb{E}\left[\theta^{3}\right]-2 \mathbb{E}\left[\theta^{2}\right]+\mathbb{E}[\theta]}{\mathbb{E}\left[\theta^{2}\right]-2 \mathbb{E}[\theta]+1}$ \\
& $\hat{s}_{D}=s_{j}=B$ & $\frac{f(\theta) \theta^{2}}{\int_{0}^{1} f(\hat{\theta}) \hat{\theta}^{2} d \hat{\theta}}$ & $\frac{\mathbb{E}\left[\theta^{3}\right]}{\mathbb{E}\left[\theta^{2}\right]}$ \\
\hline
\end{tabular}

Table 1: Posterior beliefs and conditional expectations about $\theta$ given signaling environment

Note: In pooling equilibria $\hat{s}_{D}$ is uninformative so posterior beliefs and conditional expectations are formed based only on the politician's signal $s_{j}(G$ or $B)$. In separating equilibria, $s_{D}$ is informative so the politician incorporates both $\hat{s}_{D}$ and $s_{j}$ into her posteriors beliefs and conditional expectations. Note that the sets of signals $\left(\hat{s}_{D}=G, s_{j}=B\right)$ and $\left(\hat{s}_{D}=B, s_{j}=G\right)$ are exchangeable so can be represented by $\hat{s}_{D} \neq s_{j}$. The Moderate's policy choice is equal to the conditional expectation depending on equilibrium type and information set and Ally's analogous policy choice is equal to the conditional expectation scaled toward zero by $b$.

reveal the Donor's signal in which case the candidate updates using Bayes' rule as if she observed both signals. In this case, let $\hat{s}_{D}\left(c^{M}, c^{A}\right)$ be candidate $j$ 's estimate of $s_{D}$ given Donor contributions $\left(c^{M}, c^{A}\right)$. The candidates' beliefs at each information set in both settings are listed in Table 1 along with the conditional expectations and fully derived in Online Appendix A.1 (p. 1). In each case the expectation of $\theta$ is increasing in the number of $B$ signals observed or inferred.

\section{Policy choices}

Politician policy strategies. The previous section characterizes players' conditional expectations regarding $\theta$. We begin our analysis of players' actions with a result that characterizes candidate equilibrium policy choices as a function of these beliefs.

Lemma 1. The Moderate candidate sets policy to $\mathbb{E}\left[\theta \mid s_{M}, \hat{s}_{D}\left(c^{M}, c^{A}\right)\right]$, her expectation of $\theta$ given her signal and Donor contributions. The Ally candidate sets policy to $\mathbb{E}\left[\theta \mid s_{A}, \hat{s}_{D}\left(c^{M}, c^{A}\right)\right] / b$ or her analogous expectation of $\theta$ scaled toward zero proportional to her bias $b$. The difference in 
Moderate and Ally policy choices is therefore increasing in the Ally's bias $b$ and in the expected value of the state $\theta$. Furthermore, the Ally becomes identical to the Moderate as $b \rightarrow 1$ and becomes perfectly aligned with the Donor as $b \rightarrow \infty$.

Lemma 1 provides several immediate implications. First, information that increases the expected value of $\theta$ for both candidates also increases the difference between their optimal policies. As each candidate's expectation of $\theta$ increases, holding the Ally's bias fixed, the Moderate's optimal policy shifts more than the Ally's does. Second, the difference between the optimal policies of the Moderate and the Ally also increases as the Ally's bias $b$ increases. As $b$ approaches its lower bound of one, there is no difference between the Moderate and the Ally. As $b$ grows arbitrarily large, the Ally's optimal policy for any beliefs goes to zero, implying that the Ally's preferences approach those of the Donor.

Lemma 1 also allows us to fully describe candidate policy strategies in pooling and separating equilibria. In a pooling equilibrium, the Donor's contributions are uninformative. Thus, candidates choose policy based only on their own signals: $x_{M}^{P o o l}\left(s_{M}\right)=\mathbb{E}\left[\theta \mid s_{M}\right]$ and $x_{A}^{\text {Pool }}\left(s_{A}\right)=$ $\mathbb{E}\left[\theta \mid s_{A}\right] / b$. In a separating equilibrium, the Donor's contributions are informative and reveal his signal so that $x_{M}^{S e p}\left(s_{M}, \hat{s}_{D}\left(c^{M}, c^{A}\right)\right)=\mathbb{E}\left[\theta \mid s_{M}, \hat{s}_{D}\left(c^{M}, c^{A}\right)\right]=\mathbb{E}\left[\theta \mid s_{M}, s_{D}\right]$ and $x_{A}^{S e p}\left(s_{A}, \hat{s}_{D}\left(c^{M}, c^{A}\right)\right)=$ $\mathbb{E}\left[\theta \mid s_{A}, \hat{s}_{D}\left(c^{M}, c^{A}\right)\right] / b=\mathbb{E}\left[\theta \mid s_{A}, s_{D}\right] / b$. This implies that in a pooling equilibrium, each candidate pursues one of only two distinct policy choices: one for each value of their signal. In contrast, in a separating equilibrium, candidate policy choices respond to both their own signals and those revealed by the Donor. Though their are four combinations of signals in that case, exchangeability of the signals means that candidates respond to the sum of the two signals and, therefore, the candidates have only three distinct policy choices.

Expected policy from the Donor's perspective. We can understand the politicians' choices from Lemma 1 differently by considering expected policies from the perspective of the Donor. When politicians act as if the Donor's contributions reveal his private information, the expected policies from each politician from the perspective of a type $s_{D}$ Donor whose contributions induce 
the belief that he is type $\hat{s}_{D}$ are given by,

$$
\begin{aligned}
\mathbb{E}\left[x_{M}^{S e p} \mid \hat{s}_{D}, s_{D}\right] & =\operatorname{Pr}\left[s_{M}=B \mid s_{D}\right] x_{M}^{S e p}\left(B, \hat{s}_{D}\right)+\operatorname{Pr}\left[s_{M}=G \mid s_{D}\right] x_{M}^{S e p}\left(G, \hat{s}_{D}\right), \\
& =\mathbb{E}\left[\theta \mid s_{D}\right] \mathbb{E}\left[\theta \mid s_{M}=B, \hat{s}_{D}\right]+\left(1-\mathbb{E}\left[\theta \mid s_{D}\right]\right) \mathbb{E}\left[\theta \mid s_{M}=G, \hat{s}_{D}\right], \text { and } \\
\mathbb{E}\left[x_{A}^{S e p} \mid \hat{s}_{D}, s_{D}\right] & =\mathbb{E}\left[x_{M}^{S e p} \mid \hat{s}_{D}, s_{D}\right] / b,
\end{aligned}
$$

where the move from the first line to the second follows from Lemma 1 and the fact that $\operatorname{Pr}\left[s_{M}=\right.$ $\left.B \mid s_{D}\right]=\mathbb{E}\left[\theta \mid s_{D}\right]$. Because the politicians' signals are positively correlated with the true $\theta$ and the Donor's information causes them to update their beliefs about $\theta$, the good type of Donor expects a more favorable (lower) policy from either politician than does the bad type even if both types sent the same signal with their contributions (i.e. $\mathbb{E}\left[x_{M}^{S e p} \mid \hat{s}_{D}, G\right]<\mathbb{E}\left[x_{M}^{S e p} \mid \hat{s}_{D}, B\right]$ and $\mathbb{E}\left[x_{A}^{S e p} \mid \hat{s}_{D}, G\right]<$ $\mathbb{E}\left[x_{A}^{S e p} \mid \hat{s}_{D}, B\right]$ for any $\left.\hat{s}_{D}\right)$. This shows how different types of Donors may have different signaling incentives even though they have the same policy preferences and their contributions could have similar effects.

\section{Informative campaign contributions}

Having established politicians' policy choices given their beliefs, we now derive conditions under which campaign contributions reveal the Donor's private information. In a typical signaling model this would require either policy preferences or costs of some contributions to vary by Donor type. This is not the case in our model. Instead, favorable conditions for information transmission arise endogenously because of a combination of Donor information, politician information, and electoral effects of contributions.

A separating equilibrium takes the following form. All bad types choose a contribution schedule $\left(c_{B}^{M}, c_{B}^{A}\right)$. All good types choose a different contribution schedule $\left(c_{G}^{M}, c_{G}^{A}\right)$ with the property that $p\left(c_{G}^{M}, c_{G}^{A}\right)>p\left(c_{B}^{M}, c_{B}^{A}\right)$. That is, the electoral effect of the good type's contribution is to increase the likelihood of electing the Moderate relative to the bad type's contribution. The politicians believe that the Donor is a bad type if they observe any contributions for which $p\left(c^{M}, c^{A}\right)<p\left(c_{G}^{M}, c_{G}^{A}\right)$ and otherwise believe the Donor is a good type. In particular, this tells us that $\hat{s}\left(c_{B}^{M}, c_{B}^{A}\right)=B$, and 
$\hat{s}\left(c_{G}^{M}, c_{G}^{A}\right)=G$ so that contributions perfectly reveal the Donor's private information on the equilibrium path of play. Furthermore, given that any contributions such that $p\left(c^{M}, c^{A}\right)<p\left(c_{G}^{M}, c_{G}^{A}\right)$ induce the belief that the Donor is a bad type, the rational contribution for the bad type of Donor in a separating equilibrium maximizes favorable electoral effects so we must have $\left(c_{B}^{M}, c_{B}^{A}\right)=(0,1)$.

To show that this is an equilibrium we must show that, given rational policymaking strategies by both politicians, the donation strategy is incentive compatible in the sense that bad types indeed prefer $\left(c_{B}^{M}, c_{B}^{A}\right)$ and good types prefer $\left(c_{G}^{M}, c_{G}^{A}\right)$. A Donor of type $s_{D}$ prefers to give $\left(c_{G}^{M}, c_{G}^{A}\right)$ over $\left(c_{B}^{M}, c_{B}^{A}\right)=(0,1)$ if,

$$
\begin{array}{r}
-p\left(c_{G}^{M}, c_{G}^{A}\right) \mathbb{E}\left[x_{M}^{S e p} \mid \hat{s}_{D}=G, s_{D}\right]-\left(1-p\left(c_{G}^{M}, c_{G}^{A}\right)\right) \mathbb{E}\left[x_{M}^{S e p} \mid \hat{s}_{D}=G, s_{D}\right] / b> \\
-p(0,1) \mathbb{E}\left[x_{M}^{S e p} \mid \hat{s}_{D}=B, s_{D}\right]-(1-p(0,1)) \mathbb{E}\left[x_{M}^{S e p} \mid \hat{s}_{D}=B, s_{D}\right] / b
\end{array}
$$

On the left-hand side of Inequality (7), the first term captures the Donor's utility in the event the Moderate candidate wins office and the second term captures the Donor's utility when the Ally candidate wins office given the effects contributions $\left(c_{G}^{M}, c_{G}^{A}\right)$ have on the electoral outcome as well as the politicians' beliefs. On the right-hand side of Inequality (7) we have the same calculation given $\left(c_{B}^{M}, c_{B}^{A}\right)=(0,1)$ so that when the inequality holds the Donor strictly prefers to give $\left(c_{G}^{M}, c_{G}^{A}\right)$. In a separating equilibrium Inequality (7) is satisfied for good types but not for bad types.

Rearranging the incentive compatibility condition in Inequality (7) yields,

$$
\underbrace{\frac{\mathbb{E}\left[x_{M}^{S e p} \mid \hat{s}_{D}=G, s_{D}\right]}{\mathbb{E}\left[x_{M}^{S e p} \mid \hat{s}_{D}=B, s_{D}\right]}}_{\text {Persuasion effects }}<\underbrace{\frac{p(0,1)(b-1)+1}{p\left(c_{G}^{M}, c_{G}^{A}\right)(b-1)+1}}_{\text {Electoral effects }},
$$

which separates the electoral effects of contributions from the persuasion effects. The left-hand side of the inequality captures how much the Donor benefits on policy grounds from inducing the politicians to believe that he received a good signal instead of a bad one, where smaller ratios indicate a larger benefit since the Donor values policy closer to zero. As the persuasion effects ratio approaches zero the incentive to contribute $\left(c_{G}^{M}, c_{G}^{A}\right)$ and induce the belief $\hat{s}_{D}=G$ is strengthened 
because doing so leads to more favorable policy from the Donor's perspective. In contrast, as the ratio approaches one there are small policy differences across the different induced beliefs, which weakens the incentive to persuade. The right-hand side of Inequality (8) captures the total electoral effect of contributions, incorporating their effect on election outcomes and weighing them more heavily for higher levels of polarization where electoral outcomes are more consequential. If contributions have no effect or there is no polarization (i.e., $p$ is constant or $b=1$ ) then this ratio is equal to one, indicating no electoral effects. As electoral effects of contributions become large (i.e., $p(0,1)$ goes to 0 and $p\left(c_{G}^{M}, c_{G}^{A}\right)$ goes to 1 and $\left.b \rightarrow \infty\right)$ the right-hand side of Inequality (8) goes to zero indicating that electorally-motivated contributions have large returns.

To show the possibility of a separating equilibrium we must demonstrate that the model exhibits a single-crossing condition which here means that if Inequality (8) holds weakly for a bad type of Donor then it must hold strictly for a good type. More broadly this implies that a good Donor has a stronger incentive to choose $\left(c_{G}^{M}, c_{G}^{A}\right)$ in order to signal that his type is $G$. Since the persuasion effects in Inequality (8) depend on the Donor's type $s_{D}$ and the electoral effects do not, the singlecrossing condition is equivalent to showing that the persuasion effects are ordered:

$$
\frac{\mathbb{E}\left[x_{M} \mid \hat{s}_{D}=G, s_{D}=G\right]}{\mathbb{E}\left[x_{M} \mid \hat{s}_{D}=B, s_{D}=G\right]}<\frac{\mathbb{E}\left[x_{M} \mid \hat{s}_{D}=G, s_{D}=B\right]}{\mathbb{E}\left[x_{M} \mid \hat{s}_{D}=B, s_{D}=B\right]}
$$

Before establishing the general result we turn to a parametric example to illustrate why this inequality holds.

Example 1. Consider an example in which $\theta$ is uniformly distributed on the unit interval. In this case, the Donor's expectation of $\theta$ conditional on a good signal is $\frac{1}{3}$, compared to an expectation of $\frac{2}{3}$ following a bad signal. Furthermore, the Moderate's optimal policy is $\frac{3}{4}$ if she believes that both signals were bad, $\frac{1}{2}$ if she believes that one signal was bad, and $\frac{1}{4}$ if she believes that both signals were good. ${ }^{5}$ As a result, the Donor's absolute expected policy improvement from con-

\footnotetext{
${ }^{5}$ These calculations all follow from recalling that a uniform distribution is equivalent to a Beta $(1,1)$ and that the correct posterior belief is $\operatorname{Beta}\left(1+n_{B}, 1+n_{s}-n_{B}\right)$ (where $n_{s}$ is the total number of signals and $n_{B}$ is the number of bad signals) and then applying the formula for the expectation of a Beta distributed random variable.
} 
vincing the Moderate he is a good type rather than a bad type is always equal to $\frac{1}{4}$ in the uniform model: the policy either moves from $\frac{3}{4}$ to $\frac{1}{2}$ when $s_{M}=B$ or from $\frac{1}{2}$ to $\frac{1}{4}$ when $s_{M}=G$. However, when weighing this persuasive benefit against changes in the probability of electing the Moderate the Donor considers relative rather than absolute changes. Therefore the difference in incentives between types comes instead from baseline differences in the probability of each politician signal. From the Donor's perspective, the probability that the politician's signal is bad is equal to the posterior expectation of $\theta$. Therefore, the Donor believes that the winning politician's signal will match his own signal with probability $\frac{2}{3}$. This correlation in signals means that the good type of Donor expects more favorable policy from politicians than does the bad type even if both types induced the same politician beliefs. Consequently, the same absolute decrease in $x$ is a larger relative decrease for the good type. Another way of thinking about this comparison is to note that the expected policy difference between the Moderate and Ally is smaller for the good type than for the bad type. Either way this illustrates why good types are more willing to increase the risk of electing the Moderate in order to persuade policymakers.

Lemma 2 establishes that the relative benefit of persuasion is higher for good types not just in the uniform model but for a general prior distribution of $\theta$.

Lemma 2. For any prior belief $f$ we have,

$$
\frac{\mathbb{E}\left[x_{M} \mid \hat{s}_{D}=G, s_{D}=G\right]}{\mathbb{E}\left[x_{M} \mid \hat{s}_{D}=B, s_{D}=G\right]}<\frac{\mathbb{E}\left[x_{M} \mid \hat{s}_{D}=G, s_{D}=B\right]}{\mathbb{E}\left[x_{M} \mid \hat{s}_{D}=B, s_{D}=B\right]} .
$$

Lemma 2 establishes single crossing which opens the door to informative campaign contributions by establishing that a good Donor indeed has a stronger incentive to signal his type. The remaining issue is whether there exists a contribution with large enough electoral effects to deter bad types of Donors from imitating good types. To establish a necessary condition for deterring imitation we appeal to a simple argument using the intermediate value theorem. First, we check whether the bad type of Donor would be willing to give his entire contribution budget to the Moderate in order to persuade politicians that he is a good type. If the bad type of Donor would not be 
deterred by this extreme contribution then he would not be deterred by any other contribution either and there cannot be a separating equilibrium. If the bad type of Donor would be unwilling to contribute his entire budget to the Moderate in order to imitate the good type then, since the difference between the persuasion effect and the electoral effect is a continuous function of the contribution amounts, there must be some less extreme choice of $\left(c_{G}^{M *}, c_{G}^{A *}\right)$ with $p(0,1)<p\left(c_{G}^{M *}, c_{G}^{A *}\right) \leq p(1,0)$ that would make the bad type indifferent between imitating or not. The single-crossing condition implies that the good type strictly prefers to give this $\left(c_{G}^{M *}, c_{G}^{A *}\right)$ so this is a separating equilibrium.

Proposition 1. There is a separating equilibrium if

$$
\frac{\mathbb{E}\left[x_{M}^{S e p} \mid \hat{s}_{D}=G, s_{D}=B\right]}{\mathbb{E}\left[x_{M}^{S e p} \mid \hat{s}_{D}=B, s_{D}=B\right]} \geq \frac{p(0,1)(b-1)+1}{p(1,0)(b-1)+1} .
$$

In a separating equilibrium the bad type of Donor contributes his entire budget to the Ally and the good type of Donor's contribution increases the probability of electing the Moderate enough to deter imitation by bad types.

Some additional implications follow immediately from the condition in Proposition 1. The first is that in order to support separating equilibrium through campaign contributions there must exist substantial enough electoral effects as well as meaningful preference divergence between candidates. This is illustrated by inspecting the electoral effect in Proposition 1. As $b$ converges to one (no preference divergence) or as $p(1,0)$ and $p(0,1)$ converge to some common probability (no electoral effects), the ratio of the electoral effects goes to one, which means that any signaling benefit would induce a deviation from the bad type of Donor. Remark 1 states this result.

Remark 1. Separating equilibria do not exist if the preferences of the candidates are too similar (i.e., b close to 1) or as the electoral effect of contributions goes to zero.

Another implication of Proposition 1 allows us to categorize equilibria by examining the relative size of the electoral and persuasion effects. Most obviously, if the condition from Proposition 1 fails (i.e., the hypothetical persuasion effects dominate the electoral effects) then any equilibrium 
is a pooling equilibrium. Since the motivations for the Donor in a pooling equilibrium are typically purely electoral, both types of Donor will maximize their electoral impact by giving their entire budget to the Ally. Additionally, we can consider a slight modification of the electoral condition to consider two different patterns of contributions we may observe in a separating equilibrium. If a stronger version of the conditions in Proposition 1 held and the bad type of Donor would not even be willing to reduce his contributions to zero in order to persuade the politicians, a separating equilibrium may exist in which the good type of Donor signals simply by reducing his contributions to the Ally, which we call persuasion by moderation. If this stronger condition does not hold but the conditions in Proposition 1 do hold, then the equilibrium behavior must involve good types contributing to the Moderate, which we refer to as persuasion by switching sides.

Proposition 2. Equilibria to the game fall in one of three categories:

- (Persuasion by moderation) If the bad type would not strictly prefer to reduce contributions to zero in order to persuade the politicians then there is a separating equilibrium in which the good type of Donor reduces his contribution to the Ally without contributing to the Moderate

- (Persuasion by switching sides) If the bad type would reduce contributions to zero in order to persuade the politicians but would not give his whole budget to the Moderate then there is a separating equilibrium in which the good type of Donor contributes to the Moderate.

- (Pure electoral motivation) If neither of the aforementioned conditions hold, any equilibrium is a pooling equilibrium.

Though other pooling equilibria are possible in the third part of Proposition 2, we argue that the strongest prediction in this situation is one in which both types of Donor only give to the Ally. This is true for two reasons. First, this allows us to capture the situation in which donors give in a way that is consistent with a model including only electoral motivations. Thus, we see this as the most empirically realistic prediction. Second, if there exists more than one pooling equilibrium, the best one for the Donor is the one that allows her to allocate her contributions to the Ally. For both of 
these reasons, we equate a pooling equilibrium with pure electoral motivation in our discussion of empirical implications.

\section{Costly contributions}

The baseline model assumes no direct cost of contributions. This omission is instructive because it illustrates one contrast between our model and other signaling models in the campaign finance literature: the signaling value of campaign finance in our theory comes from the campaign rather than the finances. But of course, in reality, contributions are costly. To ensure that the model's insights are broadly applicable we consider an extension of the model in which contributions also have a direct financial cost. The extended model makes more realistic predictions about the pattern of contributions (e.g., bad types of Donors do not always spend their entire budget), but our main argument holds true in the presence of direct costs. Direct costs of contributions do not significantly alter the mechanisms supporting separating equilibria because these direct costs, unlike the endogenous, indirect costs described above, do not depend on the Donor's type. Specifically, we show that contributions that are more friendly to the Moderate candidate signal favorable information as in the baseline model and that, even in the presence of direct costs, meaningful separating equilibria only exist when there is enough preference divergence between candidates and contributions have large enough electoral effects.

The extended model is similar to the baseline model except that the Donor's utility function incorporates a direct cost of contributions. For simplicity we represent these costs as a fixed marginal cost $k>0$, though the analysis easily extends to cases in which the cost is not linear. The Donor's utility function is therefore,

$$
u_{D}\left(x, c^{M}, c^{A}\right)=-x-k\left(c^{M}+c^{A}\right)
$$

The addition of another parameter limits the tractability of the model under the general distributional assumptions on $\theta$ described in the baseline model so we will also add the assumption that 
prior beliefs about $\theta$ follow a Beta distribution with shape parameters $\alpha>0$ and $\beta>0 .^{6}$

The most important consequence of including direct financial costs is that electoral motivations may not drive donors to contribute their entire budget to their preferred candidates. Any differences between the costly contributions model and the baseline model follow from this fact. In fact, if costs are small enough that the bad type of Donor is willing to spend his entire budget on electing the Ally, the conditions needed to support a separating equilibrium are identical to the baseline. Otherwise, we need a way to characterize interior solutions to the electorally motivated Donor's optimization problem. To that end, we assume that $p\left(c^{M}, c^{A}\right)$ is twice differentiable and that $\frac{\partial^{2} p\left(0, c^{A}\right)}{\partial c^{A^{2}}}>0$ for all $c^{A} \geq 0$. The assumptions from the baseline model additionally imply that $\frac{\partial p\left(c^{M}, c^{A}\right)}{\partial c^{M}}>0$ and $\frac{\partial p\left(c^{M}, c^{A}\right)}{\partial c^{A}}<0$.

Proposition 3 lists the main result for the model with costly contributions. The upshot is that the addition of direct contribution costs does not significantly change the conditions required to support a separating equilibrium to the game. First, the sorting condition of Lemma A.2 still holds in the costly contributions game because the direct costs do not depend on whether the Donor is a good type or bad type. Second, even with costly contributions, separating equilibria in this model cannot exist without meaningful electoral costs, illustrating that the electoral and informational effects remain the driving forces in this model. Finally, the conditions determining whether or not a separating equilibrium exists are derived in the same manner as in the baseline model: We ask whether the bad type of Donor, in order to persuade the politicians, would willingly contribute his entire budget to the Moderate rather than give his default contribution. If not, we conclude that there must be a separating equilibrium, since some contribution would deter the bad type of Donor from imitating and the good type of Donor is strictly more willing to help the Moderate. The only thing the addition of costs changes is the value of the bad type's default contribution.

Proposition 3. Let $c^{*}$ be the optimal contribution to the Ally for the bad type of Donor in the

\footnotetext{
${ }^{6}$ As we discuss in the Online Appendix (pp. 5-7), the results do not rely on the Beta prior when $k$ is not too large but a full characterization of equilibria relies on this distributional assumption.
} 
absence of signaling incentives. If

$\mathbb{E}\left[x_{M}^{S e p} \mid \hat{s}_{D}=B, s_{D}=B\right] \frac{p\left(0, c^{*}\right)(b-1)+1}{b}-\mathbb{E}\left[x_{M}^{S e p} \mid \hat{s}_{D}=G, s_{D}=B\right] \frac{p(1,0)(b-1)+1}{b} \leq k\left(c^{M}+c^{A}-c^{*}\right)$

then there exists a separating equilibrium in which the bad type of Donor contributes $\left(0, c^{*}\right)$ and the good type of Donor contributes some $\left(\tilde{c}^{M}, \tilde{c}^{A}\right)$ with $p\left(\tilde{c}^{M}, \tilde{c}^{A}\right)>p\left(0, c^{*}\right)$. There is no separating equilibrium if b is too close to one or if the electoral effects of contributions are too small.

Proposition 3 should not be taken to mean that costs do not matter at all. They change the pattern of contributions to be more realistic and, in one important way, improve the chances for a separating equilibrium. The way costs might enhance the possibility of information transmission is by further reducing the bad type of Donor's utility for imitating. If the bad type's default contribution is relatively small, a large contribution to the Moderate may help deter imitation by bad types while the electoral and informational effects described above ensure that the good type of Donor is more willing to pay.

\section{Discussion of empirical implications}

Electoral effects and contribution behavior. One implication of our model is that contributions are informative when their electoral effects are large enough. We may therefore observe differences between close elections and landslide elections with respect to strategic contribution behavior.

However, this result may raise questions. Since the effect of any one contribution is likely to be small, is it really plausible that contributions could generate credible information through this mechanism? We think so. It is important to keep in mind that electoral effects are always evaluated relative to policy effects in this model. We expect that the policy effect of any one contributor's behavior is also relatively small for many reasons: there are many other donors, there may be well established policy opinions (corresponding to precise priors in the model), and so on.

Our condition for separating equilibria in Proposition 1 means that the disadvantage the Donor incurs on policy by not imitating the good type is no larger than the maximum electoral disadvan- 
tage created by giving to the less-preferred candidate. However, this does not imply that contributions must have an unrealistically large impact on electoral outcomes. One reason is that, on a typical issue, policymakers ought to have reasonably well-informed priors which means that the scope for policy movement due to persuasion is relatively narrow. Consider, for instance, regulation of coal fired power plants. A value of $\theta=0$ would mean that coal powered plants present no risks whatsoever and should be completely unregulated while a value of $\theta=1$ would mean that the risks are so urgent that they should be completely shut down. In reality, the scope of debate is much narrower, involving the rate at which old technology should be updated, marginal changes to emissions requirements, and so on. This in turn decreases the size of the policy effect of contributions while leaving electoral effects unaltered, which means a separating equilibrium may be sustained with relatively small electoral effects. Online Appendix B.1 (pp. 8-10) argues that separating equilibria can be supported with realistic electoral effects given moderately precise priors.

We also consider a situation in which there are many identical donors. We may think of this as an extension of the model to small donors. Ansolabehere, de Figueiredo and Snyder (2003) note the importance of small contributions and use them to argue that most donations are driven by non-instrumental factors. In this situation the size of the electoral effects and the size of the policy effects each diminish with the number of Donors. Nevertheless, we show that a separating equilibrium can still be sustained as the number of donors grows large.

Example 2 in Online Appendix B.1.1 (pp. 10-13) uses a numerical example to demonstrate that separating equilibria may still exist as the number of donors increases. The key issue is how quickly persuasion effects go to zero, relative to electoral effects, as the number of donors gets large. If electoral effects diminish very quickly relative to persuasion effects - that is, if the electoral effect of contributions diminishes very quickly but the persuasion effect of an extra signal does not then separating equilibria will diminish more quickly as the number of donors becomes larger. Conversely, if the electoral effect of contributions diminishes at approximately the same rate (or slower) than the persuasive effect of contributions as the number of donors grows then separating 
equilibria will still exist in the way we have described above.

One caveat is that both of these examples return to the baseline case in which contributions are not costly. As the electoral and policy effects of contributions each go to zero few people should be willing to make costly contributions at all. This is a good baseline prediction since less than one half of one percent of citizens give any itemized campaign donations (Donor Demographics N.d.). However, it indicates that when looking at donations we are dealing with a selection of individuals with a very small cost of donating.

Patterns of contributions. One way our model adds to the campaign finance literature is by offering predictions about the patterns of contributions across parties and candidates. Brunell (2005) offers a rich description of PAC contribution behavior that is consistent with a flexible interpretation of our model. Brunell argues that each PAC has a clear preference about which party controls Congress though they also contribute across party lines at times. Additionally, our findings are consistent with evidence suggesting that corporations and trade groups are more politically conservative than we would infer if we assumed all contributions were expressions of sincere electoral preferences (Thieme 2020; Grumbach and Pierson 2020). More broadly, these observations suggest the need for more theorizing about when and why groups give across the aisle and our model represents a step in that direction.

Returns to backing a winner. According to recent research there is no evidence of good returns to backing the person who ultimately wins the election (Fowler, Garro and Spenkuch 2019). This finding runs counter to the prediction of a pure electoral model or a quid pro quo model of contributions but does not refute signaling models such as this one. In some of our separating equilibria the good type of Donor may expect to be better off if they back the candidate who loses the election, since they still benefit from the persuasion effect of backing the Moderate without the cost of the Moderate actually winning office. In other cases, the returns to backing a winner may be positive. The average return may therefore be close to zero, though the average masks some unobserved heterogeneity between Donors. Thus, the average effect of contributions may be zero even though some donors actually do receive some positive returns to backing a winner. 
Causal effect of contributions and policy decisions. Though citizens and commentators widely suspect that legislators' policy choices are influenced by campaign contributions, social scientists have not found much evidence that contributions affect votes on policy. For instance, Ansolabehere, de Figueiredo and Snyder (2003) reviewed the literature and concluded that, in three out of four cases, contributions either had no significant effects or they were in the wrong direction (114). Though our model predicts that contributions can influence politicians' policy choices, that influence depends on two factors that researchers may not observe: the game's equilibrium and Donor type.

We consider what results would be found by accurately estimating the causal effect of contributions from the Donor on roll call votes (or some other policy choice). This would measure, for an individual politician, the decisions in favor of the Donor's policy given contributions from the Donor minus the decision in favor of the Donor given no, or lower levels of, contributions from the Donor. Table 2 summarizes the possibilities. In a pooling equilibrium, contributions should have no effect on votes since they convey no information to the candidates. In a persuasion by moderation equilibrium, contributions go to allies of the Donor and may have opposite effects depending on their size: "restrained" contributions in smaller amounts have a favorable effect on policy choices from the Donor's perspective. Thus, a regression of policy choices on contribution amounts would find effects running in the opposite direction to that desired. In a persuasion by switching sides equilibrium, only bad types of donors contribute to allies and these contributions have the "wrong sign" (i.e., contributions would appear to be associated with policy choices unfavorable to the Donor). Contributions to opponents, which signal that the Donor is a good type, have the usual expected effect on policy choice. Thus, if researchers are missing critical information about the model parameters and do not consider what information the donors may possess, it is easy to understand why even studies with otherwise sound research designs would not find consistent evidence of policy influence.

To compound matters, the effects of contributions on policy choices in our model apply to both candidates, not just the recipient. In fact, all effects of contributions in our model are more 


\begin{tabular}{lll}
\hline Equilibrium (type) & Contribution & Predicted Effect of Contributions \\
\hline Pooling (any type) & Allies & Zero \\
Restraint (bad type) & Allies & Unfavorable to Donor \\
Restraint (good type) & Allies & Favorable to Donor \\
Switching sides (bad type) & Allies & Unfavorable to Donor \\
Switching sides (good type) & Opponents (Moderate) & Favorable to Donor \\
\hline
\end{tabular}

Table 2: Predicted causal effect of contributions on a candidate's policy choice for each equilibrium and type of donor.

pronounced when the Moderate wins the election, regardless of which candidate receives the contributions. Thus, we would expect empirical designs to find little or no effects of contributions in cross-sectional comparisons between legislators who receive contributions and those who do not. Analyses controlling for legislator fixed effects adjust for this by controlling for the type of legislator (Moderate versus Ally) but the average effect ignores interesting heterogeneity in treatment effects across different legislators.

\section{Conclusion}

In this paper, we studied how campaign donors, such as an interest group, trade association, or individual "mega-donors" might use campaign contributions to either persuade policymakers to choose policies in line with their interests or influence an election outcome. Our argument shows that campaign contributions as informative signals that alter politician behavior are fundamentally tied to the effect of those contributions on electoral prospects. Importantly, this dynamic does not depend on the costs of political expenditures per se, as in much of the previous work on the topic, but it does depend on endogenous costs that arise due to interaction of the electoral and informational effects of contributions. Thus, our theory points out the value of campaign contributions specifically, relative to other political expenditures.

In addition, our results provide insight into several empirical questions in the study of campaign finance. Our theory aids in understanding patterns of giving in which donors contribute to politicians or parties that hold opposing viewpoints. It also provides insight into why donors may benefit from donations to opposing candidates. We also provide insight into why returns from 
contributions and the relationship between contributions and roll call votes may be difficult to uncover given what analysts normally observe, as well as novel predictions about what circumstances may allow us to uncover causal effects of interest. Finally, we show that when multiple avenues of influence are available to interest groups, the choice of one avenue may lead to informational spillovers in others. This insight may apply beyond the current context of campaign finance to lobbying, endorsements, and public relations campaigns among other things. Ultimately, our theory suggests that to address the myriad questions about campaign finance we must account for how informational effects of financing are fundamentally intertwined with effects on the campaign.

\section{References}

Ansolabehere, Stephen, John M. de Figueiredo and James M. Snyder. 2003. "Why Is There so Little Money in U.S. Politics?” Journal of Economic Perspectives 17(1):105-130.

Arceneaux, Kevin and Robin Kolodny. 2009. "Educating the Least Informed: Group Endorsements in a Grassroots Campaign.” American Journal of Political Science 53(4):755-770.

Ashworth, Scott. 2006. "Campaign finance and voter welfare with entrenched incumbents." American Political Science Review 100(1):55-68.

Austen-Smith, David. 1995. "Campaign Contributions and Access.” American Political Science Review 89(3):566-581.

Austen-Smith, David. 1998. “Allocating Access for Information and Contributions.” Journal of Law, Economics, and Organization 14(2):277-303.

Barber, Michael. 2016. "Donation Motivations: Testing Theories of Access and Ideology.” Political Research Quarterly 69(1):148-159.

Barber, Michael J., Brandice Canes-Wrone and Sharece Thrower. 2017. "Ideologically Sophisticated Donors: Which Candidates Do Individual Contributors Finance?" American Journal of Political Science 61(2):271-288. 
Bonica, Adam. 2013. "Ideology and Interests in the Political Marketplace." American Journal of Political Science 57(2):294-311.

Brunell, Thomas L. 2005. "The Relationship Between Political Parties and Interest Groups: Explaining Patterns of PAC Contributions to Candidates for Congress." Political Research Quarterly 58(4):681-688.

Coate, Stephen. 2004. "Political Competition with Campaign Contributions and Informative Advertising." Journal of the European Economic Association 2(5):772-804.

Cotton, Christopher. 2009. "Should we tax or cap political contributions? A lobbying model with policy favors and access." Journal of Public Economics 93(7-8):831-842.

Cotton, Christopher. 2012. "Pay-to-play politics: Informational lobbying and contribution limits when money buys access." Journal of Public Economics 96.

Cotton, Christopher. 2016. "Competing for Attention: Lobbying Time-Constrained Politicians." Journal of Public Economic Theory 18(4):642-665.

Donor Demographics. N.d.

URL: https://www.opensecrets.org/overview/donordemographics.php. Last accessed: February $5,2020$.

Erikson, Robert S. and Thomas R. Palfrey. 2000. "Equilibria in Campaign Spending Games: Theory and Data." American Political Science Review 94(3):595-609.

Felli, Leonardo and Antonio Merlo. 2007. "If You Cannot Get Your Friends Elected, Lobby Your Enemies." Journal of the European Economic Association 5(2-3):624-635.

Fowler, Anthony, Haritz Garro and Jörg L. Spenkuch. 2019. "Quid Pro Quo? Corporate Returns to Campaign Contributions.” Journal of Politics Forthcoming.

URL: https://www.journals.uchicago.edu/doi/pdfplus/10.1086/707307. Last accessed: February 5, 2020 . 
Fox, Justin and Lawrence S. Rothenberg. 2011. "Influence without Bribes: A Non-Contracting Model of Campaign Giving and Policymaking." Political Analysis 19(3):325-341.

Gordon, Sanford C. and Catherine Hafer. 2005. "Flexing Muscle: Corporate Political Expenditures as Signals to the Bureaucracy." American Political Science Review 99(2):245-261.

Gordon, Sanford C. and Catherine Hafer. 2007. "Corporate Influence and the Regulatory Mandate." Journal of Politics 69(2):300-319.

Green, Donald P. and Alan S. Gerber. 2015. Get Out the Vote: How to Increase Voter Turnout. Washington, D.C.: Brookings Institution Press.

Grossman, Gene M. and Elhanan Helpman. 1994. "Protection for Sale.” American Economic Review 84(4):833-850.

Grumbach, Jake M and Paul Pierson. 2020. "Are Large Corporations Politically Moderate? Using Money in Politics to Infer the Preferences of Business." Unpublished manuscript. University of Washington .

URL: https://bit.ly/39czfAt. Last accessed: February 5, 2020.

Hall, Andrew B. 2016. "Systemic Effects of Campaign Spending: Evidence from Corporate Campaign Contribution Bans in State Legislatures." Political Science Research and Methods 4(2): $343-359$.

Hertel-Fernandez, Alexander. 2018. Politics at Work: How Companies Turn Their Workers Into Lobbyists. New York, NY: Oxford University Press.

Lohmann, Susanne. 1995. "Information, Access, and Contributions: A Signaling Model of Lobbying." Public Choice 85(3):267-284.

Meirowitz, Adam. 2008. "Electoral Contests, Incumbency Advantages, and Campaign Finance.” Journal of Politics 70(3):681-699. 
Poole, Keith T., Thomas Romer and Howard Rosenthal. 1987. "The Revealed Preferences of Political Action Committees.” American Economic Review 77(2):298-302.

Potters, Jan and Frans van Winden. 1992. "Lobbying and asymmetric information.” Public Choice 74(3):269-292.

Prato, Carlo and Stephane Wolton. 2017. "Citizens United: A Theoretical Evaluation.” Political Science Research and Methods 5(3):567-574.

Sasso, Greg and Dan Alexander. 2019. "Campaign Spending and Lobbying." Unpublished manuscript .

URL: https://bit.ly/2Q9bt0q. Last accessed: February 5, 2020.

Thieme, Sebastian. 2020. "Moderation or Strategy? Political Giving by Corporations and Trade Groups.” The Journal of Politics Forthcoming.

URL: https://doi.org/10.1086/707619. Last accessed: February 5, 2020.

Wolton, Stephane. 2020. "Lobbying, Inside and Out: How Special Interest Groups Influence Policy Choices." Unpublished Manuscript. London School of Economics .

URL: https://papers.ssrn.com/sol3/papers.cfm?abstract_id=2190685. Last accessed: February $5,2020$. 


\title{
Online Supplemental Appendix Helping Friends or Influencing Foes: Electoral and Policy Effects of Campaign Finance Contributions
}

\author{
Keith E. Schnakenberg* Ian R. Turner ${ }^{\dagger}$
}

February 2020

\section{Contents}

A Proofs of results in the paper 1

A.1 Derivation of beliefs in Table 1 and proof of Lemma 1 on policy choices . . . . . . . . . . . . 1

A.2 Single crossing proofs . . . . . . . . . . . . . . . . . . . . . 2

A.3 Proofs of main results . . . . . . . . . . . . . . . . . . . . . . 4

A.3.1 Existence result in baseline model . . . . . . . . . . . . . . . . . . . . . 4

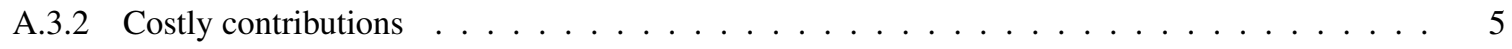

B Extensions and robustness $\quad 8$

B.1 Comparing electoral and policy effects . . . . . . . . . . . . . . . . . . 8

B.1.1 Multiple donors . . . . . . . . . . . . . . . . . . . . . 10

B.2 Separating conditions for more general Ally utility function . . . . . . . . . . . . . . . . 13

C Empirical evidence of giving to opponents 15

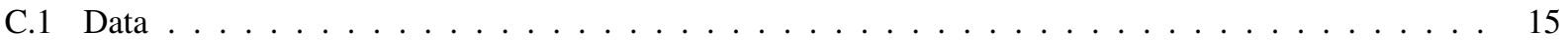

C.2 Dyad-level statistics . . . . . . . . . . . . . . . . . . . . . . . . 16

C.3 Respondent-level statistics . . . . . . . . . . . . . . . . . . . . . . . 18

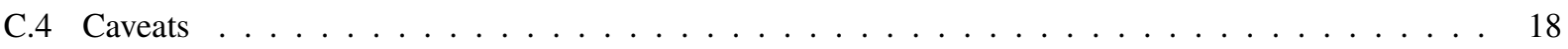

*Assistant Professor of Political Science, Washington University in St. Louis, keschnak@wustl.edu.

${ }^{\dagger}$ Assistant Professor of Political Science, Yale University, ian.turner@yale.edu. 


\section{A Proofs of results in the paper}

\section{A.1 Derivation of beliefs in Table 1 and proof of Lemma 1 on policy choices}

All players' optimal actions depend on their beliefs only through the conditional expectation of the state $\theta, \mathbb{E}[\theta \mid \cdot]$. Furthermore, these expectations, $\mathbb{E}\left[\theta \mid s_{D}\right]$ and $\mathbb{E}\left[\theta \mid s_{j}, \hat{s}_{D}\left(c^{M}, c^{A}\right)\right]$, are increasing in bad signals observed or inferred: $\mathbb{E}[\theta \mid B]>\mathbb{E}[\theta \mid G]$ and $\mathbb{E}[\theta \mid B, B]>\mathbb{E}[\theta \mid G, B]=\mathbb{E}[\theta \mid B, G]>\mathbb{E}[\theta \mid G, G]$ respectively. The fact that choices only depend on beliefs through the conditional expectations follows from the proofs of Lemma 1 (in the case of candidate choices) and Proposition 1 (in the case of the Donor) and in-text analysis. The conditional expectations that appear in Table 1 can be expressed in terms of the first three raw moments of $f$ (which all exist since the support is bounded by $[0,1]$ ) in the following way:

$$
\begin{aligned}
\mathbb{E}[\theta \mid G] & =\int_{0}^{1} \frac{f(\theta)(1-\theta) \theta}{\int_{0}^{1} f(\hat{\theta})(1-\hat{\theta}) d \hat{\theta}} d \theta=\frac{1}{1-\mathbb{E}[\theta]} \int_{0}^{1} f(\theta)(1-\theta) \theta d \theta=\frac{\mathbb{E}[\theta]-\mathbb{E}\left[\theta^{2}\right]}{1-\mathbb{E}[\theta]} \\
\mathbb{E}[\theta \mid B] & =\int_{0}^{1} \frac{f(\theta) \theta^{2}}{\int_{0}^{1} f(\hat{\theta}) \hat{\theta} d \hat{\theta}} d \theta=\frac{1}{\mathbb{E}[\theta]} \int_{0}^{1} f(\theta) \theta^{2} d \theta=\frac{\mathbb{E}\left[\theta^{2}\right]}{\mathbb{E}[\theta]} \\
\mathbb{E}[\theta \mid G, B] & =\int_{0}^{1} \frac{f(\theta)(1-\theta) \theta^{2}}{\int_{0}^{1} f(\hat{\theta}(1-\hat{\theta}) \hat{\theta} d \hat{\theta}} d \theta=\frac{1}{\mathbb{E}[\theta(1-\theta)]} \int_{0}^{1} f(\theta) \theta^{2}(1-\theta) d \theta=\frac{\mathbb{E}\left[\theta^{2}\right]-\mathbb{E}\left[\theta^{3}\right]}{\mathbb{E}[\theta]-\mathbb{E}\left[\theta^{2}\right]} \\
\mathbb{E}[\theta \mid G, G] & =\int_{0}^{1} \frac{f(\theta)(1-\theta)^{2} \theta}{\int_{0}^{1} f\left(\hat{\theta}(1-\hat{\theta})^{2} d \hat{\theta}\right.} d \theta=\frac{1}{\mathbb{E}\left[(1-\hat{\theta})^{2}\right]} \int_{0}^{1} f(\theta)(1-\theta)^{2} \theta d \theta=\frac{\mathbb{E}\left[\theta^{3}\right]-2 \mathbb{E}\left[\theta^{2}\right]+\mathbb{E}[\theta]}{\mathbb{E}\left[\theta^{2}\right]-2 \mathbb{E}[\theta]+1} \\
\mathbb{E}[\theta \mid B, B] & =\int_{0}^{1} \frac{f(\theta) \theta^{3}}{\int_{0}^{1} f(\hat{\theta}) \hat{\theta}^{2} d \hat{\theta}} d \theta=\frac{1}{\mathbb{E}\left[\theta^{2}\right]} \int_{0}^{1} f(\theta) \theta^{3} d \theta=\frac{\mathbb{E}\left[\theta^{3}\right]}{\mathbb{E}\left[\theta^{2}\right]} .
\end{aligned}
$$

Note that the signals are exchangeable so $\mathbb{E}[\theta \mid G, B]=\mathbb{E}[\theta \mid B, G]$. The binomial distribution with a fixed number of trials satisfies the Monotone Likelihood Ratio Property (Milgrom, 1981) so therefore we have $\mathbb{E}[\theta \mid B]>\mathbb{E}[\theta \mid G]$ and $\mathbb{E}[\theta \mid B, B]>\mathbb{E}[\theta \mid G, B]=\mathbb{E}[\theta \mid B, G]>\mathbb{E}[\theta \mid G, G]$.

We now prove Lemma 1 which establishes optimal policy choices.

Lemma 1. The Moderate candidate sets policy to $\mathbb{E}\left[\theta \mid s_{M}, \hat{s}_{D}\left(c^{M}, c^{A}\right)\right]$, her expectation of $\theta$ given her signal and Donor contributions. The Ally candidate sets policy to $\mathbb{E}\left[\theta \mid s_{A}, \hat{s}_{D}\left(c^{M}, c^{A}\right)\right] / b$ or her analogous expectation of $\theta$ scaled toward zero proportional to her bias $b$. The difference in Moderate and Ally policy choices is therefore increasing in the Ally's bias $b$ and in the expected value of the state $\theta$. Furthermore, the Ally becomes identical to the Moderate as $b \rightarrow 1$ and becomes perfectly aligned with the Donor as $b \rightarrow \infty$.

Proof of Lemma 1. We first prove the result for the Ally. A's expected utility is

$$
-b^{2} x_{A}^{2}+2 b x_{A} \mathbb{E}\left[\theta \mid s_{A}, \hat{s}_{D}\left(c^{M}, c^{A}\right)\right]-\mathbb{E}\left[\theta^{2} \mid s_{A}, \hat{s}_{D}\left(c^{M}, c^{A}\right)\right] .
$$


This is concave and the first-order condition with respect to $x_{A}$ is $2 b\left(\mathbb{E}\left[\theta \mid s_{A}, \hat{s}_{D}\left(c^{M}, c^{A}\right)\right]-b x_{A}\right)=0$, which gives $x_{A}=\mathbb{E}\left[\theta \mid s_{A}, \hat{s}_{D}\left(c^{M}, c^{A}\right)\right] / b$. Setting $b=1$ yields the result for $M$. Finally, comparative statics with respect to $b$ and the conditional expectations yield the final two statements in the result.

\section{A.2 Single crossing proofs}

In this section we establish the general single crossing result for the baseline model with costless contributions. We must first establish some technical results that are necessary to extend the result to a general prior distribution $f$. The following lemma is essential to the analysis.

Lemma A.1. $\mathbb{E}\left[\theta \mid s_{D}=B, s_{M}=G\right]^{2} \geq \mathbb{E}\left[\theta \mid s_{D}=s_{M}=G\right] \mathbb{E}\left[\theta \mid s_{D}=s_{M}=B\right]$.

This Lemma is straightforward to prove for a given distribution. For instance, if $f$ is a Beta distribution with parameters $\alpha$ and $\beta$, the expectations are $\frac{\alpha+\mathbb{I}_{\left(s_{D}=B\right)}+\mathbb{I}_{\left(s_{M}=B\right)}}{\alpha+\beta+2}$ and we have

$$
\left(\frac{\alpha+1}{\alpha+\beta+2}\right)^{2}-\frac{\alpha}{\alpha+\beta+2} \frac{\alpha+2}{\alpha+\beta+2}=\frac{1}{(\alpha+\beta+2)^{2}}>0
$$

A direct argument for general priors is more difficult. We start by noting that, by Theorems 3.1 and 2.6 of Dalal and Hall 1983 and by the fact that the Beta distribution is conjugate to the binomial distribution which generates our signals, any continuous prior can be arbitrarily closely approximated (in terms of total variation) by a mixture of Beta distributions. Thus, for any $\varepsilon>0$ there exists a mixture distribution $Q$ on $\mathbb{R}_{+}^{2}$ such that, letting $\phi(\theta \mid \alpha, \beta)$ denote the beta density and denoting the mixture by

$$
f_{Q}(\theta)=\int \phi(\theta \mid \alpha, \beta) d Q(\alpha, \beta)
$$

with $F_{Q}$ as the corresponding cdf,

$$
\left\|F-F_{Q}\right\|_{T V}<\varepsilon
$$

Furthermore, the posterior expectations taken with respect to $F_{Q}$ are mixtures of Beta expectations. We use this fact to prove Lemma A.1 below.

Proof of Lemma A.l. For any measure $F^{\prime}$ let $H_{F^{\prime}}=\mathbb{E}_{F^{\prime}}\left[\theta \mid s_{D}=B, s_{M}=G\right]^{2}-\mathbb{E}_{F^{\prime}}\left[\theta \mid s_{D}=s_{M}=G\right] \mathbb{E}_{F^{\prime}}\left[\theta \mid s_{D}=s_{M}=B\right]$. 
We have:

$$
\begin{aligned}
H_{F_{Q}}= & {\left[\int \frac{\alpha+1}{\alpha+\beta+2} d Q(\alpha, \beta)\right]^{2}-\left[\int \frac{\alpha}{\alpha+\beta+2} d Q(\alpha, \beta)\right]\left[\int \frac{\alpha+2}{\alpha+\beta+2} d Q(\alpha, \beta)\right] } \\
= & \iint \frac{\alpha+1}{\alpha+\beta+2} \frac{\alpha^{\prime}+1}{\alpha^{\prime}+\beta^{\prime}+2} d Q(\alpha, \beta) d Q\left(\alpha^{\prime}, \beta^{\prime}\right)-\iint \frac{\alpha}{\alpha+\beta+2} \frac{\alpha^{\prime}+2}{\alpha^{\prime}+\beta^{\prime}+2} d Q(\alpha, \beta) d Q\left(\alpha^{\prime}, \beta^{\prime}\right) \\
= & \iint\left[\frac{\alpha+1}{\alpha+\beta+2} \frac{\alpha^{\prime}+1}{\alpha^{\prime}+\beta^{\prime}+2}-\frac{\alpha}{\alpha+\beta+2} \frac{\alpha^{\prime}+2}{\alpha+\beta+2}\right] d Q(\alpha, \beta) d Q\left(\alpha^{\prime}, \beta^{\prime}\right) \\
= & \iint \frac{\alpha^{\prime}}{(\alpha+\beta+2)\left(\alpha^{\prime}+\beta^{\prime}+2\right)} d Q(\alpha, \beta) d Q\left(\alpha^{\prime}, \beta^{\prime}\right)-\iint \frac{\alpha}{(\alpha+\beta+2)\left(\alpha^{\prime}+\beta^{\prime}+2\right)} d Q(\alpha, \beta) d Q\left(\alpha^{\prime}, \beta^{\prime}\right) \\
& +\iint \frac{1}{(\alpha+\beta+2)\left(\alpha^{\prime}+\beta^{\prime}+2\right)} d Q(\alpha, \beta) d Q\left(\alpha^{\prime}, \beta^{\prime}\right) \\
= & \iint \frac{1}{(\alpha+\beta+2)\left(\alpha^{\prime}+\beta^{\prime}+2\right)} d Q(\alpha, \beta) d Q\left(\alpha^{\prime}, \beta^{\prime}\right)>0 .
\end{aligned}
$$

Let $0<\varepsilon<\iint \frac{1}{(\alpha+\beta+2)\left(\alpha^{\prime}+\beta^{\prime}+2\right)} d Q(\alpha, \beta) d Q\left(\alpha^{\prime}, \beta^{\prime}\right)$. By definition of total variation:

$$
\left|H_{F_{Q}}-H_{F}\right|<\varepsilon
$$

which implies that $\mathbb{E}\left[\theta \mid s_{D}=B, s_{M}=G\right]^{2} \geq \mathbb{E}\left[\theta \mid s_{D}=s_{M}=G\right] \mathbb{E}\left[\theta \mid s_{D}=s_{M}=B\right]$

The next Lemma establishes the single crossing condition that supports separating in this game. Lemma 2. For any prior belief $f$ we have,

$$
\frac{\mathbb{E}\left[x_{M}^{S e p} \mid \hat{s}_{D}=G, s_{D}=G\right]}{\mathbb{E}\left[x_{M}^{S e p} \mid \hat{s}_{D}=B, s_{D}=G\right]}<\frac{\mathbb{E}\left[x_{M}^{S e p} \mid \hat{s}_{D}=G, s_{D}=B\right]}{\mathbb{E}\left[x_{M}^{S e p} \mid \hat{s}_{D}=B, s_{D}=B\right]}
$$

Proof. We have,

$$
\begin{array}{r}
\frac{\mathbb{E}\left[x_{M}^{S e p} \mid \hat{s}_{D}=G, s_{D}=G\right]}{\mathbb{E}\left[x_{M}^{\text {Sep }} \mid \hat{s}_{D}=B, s_{D}=G\right]}-\frac{\mathbb{E}\left[x_{M}^{S e p} \mid \hat{s}_{D}=G, s_{D}=B\right]}{\mathbb{E}\left[x_{M}^{S e p} \mid \hat{s}_{D}=B, s_{D}=B\right]}= \\
\frac{\mathbb{E}\left[x_{M}^{S e p} \mid \hat{s}_{D}=G, s_{D}=G\right] \mathbb{E}\left[x_{M}^{S e p} \mid \hat{s}_{D}=B, s_{D}=B\right]-\mathbb{E}\left[x_{M}^{S e p} \mid \hat{s}_{D}=G, s_{D}=B\right] \mathbb{E}\left[x_{M}^{S e p} \mid \hat{s}_{D}=B, s_{D}=G\right]}{\mathbb{E}\left[x_{M}^{S e p} \mid \hat{s}_{D}=B, s_{D}=G\right] \mathbb{E}\left[x_{M}^{S e p} \mid \hat{s}_{D}=B, s_{D}=B\right]} .
\end{array}
$$

Since the signal is not perfect the denominator of the expression on the right-hand side is always strictly positive, so the entire expression is negative if,

$$
\mathbb{E}\left[x_{M}^{S e p} \mid \hat{s}_{D}=G, s_{D}=G\right] \mathbb{E}\left[x_{M}^{S e p} \mid \hat{s}_{D}=B, s_{D}=B\right)-\mathbb{E}\left[x_{M}^{S e p} \mid \hat{s}_{D}=G, s_{D}=B\right] \mathbb{E}\left[x_{M}^{S e p} \mid \hat{s}_{D}=B, s_{D}=G\right]<0 .
$$


We have:

$$
\begin{array}{r}
\mathbb{E}\left[x_{M}^{S e p} \mid \hat{s}_{D}=G, s_{D}=G\right] \mathbb{E}\left[x_{M}^{S e p} \mid \hat{s}_{D}=B, s_{D}=B\right]-\mathbb{E}\left[x_{M}^{S e p} \mid \hat{s}_{D}=G, s_{D}=B\right] \mathbb{E}\left[x_{M}^{S e p} \mid \hat{s}_{D}=B, s_{D}=G\right]= \\
\mathbb{E}\left[\theta \mid s_{D}=G\right]\left(\mathbb{E}\left[\theta \mid s_{D}=B, s_{M}=G\right]^{2}-\mathbb{E}\left[\theta \mid s_{D}=s_{M}=G\right] \mathbb{E}\left[\theta \mid s_{D}=s_{M}=B\right]\right) \\
+\mathbb{E}\left[\theta \mid s_{D}=B\right]\left(\mathbb{E}\left[\theta \mid s_{D}=s_{M}=G\right] \mathbb{E}\left[\theta \mid s_{D}=s_{M}=B\right]-\mathbb{E}\left[\theta \mid s_{D}=B, s_{M}=G\right]^{2}\right) \\
=\left(\mathbb{E}\left[\theta \mid s_{D}=G\right]-\mathbb{E}\left[\theta \mid s_{D}=B\right]\right) \\
\times\left(\mathbb{E}\left[\theta \mid s_{D}=B, s_{M}=G\right]^{2}-\mathbb{E}\left[\theta \mid s_{D}=s_{M}=G\right] \mathbb{E}\left[\theta \mid s_{D}=s_{M}=B\right]\right)
\end{array}
$$

The first term $\mathbb{E}\left[\theta \mid s_{D}=G\right]-\mathbb{E}\left[\theta \mid s_{D}=B\right]<0$ (line 3) since $\mathbb{E}[\theta \mid B]>\mathbb{E}[\theta \mid G]$. Thus, the entire expression is negative since Lemma A.1 shows that $\mathbb{E}\left[\theta \mid s_{D}=B, s_{M}=G\right]^{2}-\mathbb{E}\left[\theta \mid s_{D}=s_{M}=G\right] \mathbb{E}\left[\theta \mid s_{D}=s_{M}=B\right] \geq 0$ (line 4). This proves that $\frac{\mathbb{E}\left[x_{M}^{S e p} \mid \hat{s}_{D}=G, s_{D}=G\right]}{\mathbb{E}\left[x_{M}^{S e p} \mid \hat{s}_{D}=B, s_{D}=G\right]}<\frac{\mathbb{E}\left[x_{M}^{S e p} \mid \hat{s}_{D}=G, s_{D}=B\right]}{\mathbb{E}\left[x_{M}^{S e p} \mid \hat{s}_{D}=B, s_{D}=B\right]}$.

We will find it useful to refer to the following restatement of the single-crossing result, which we call the "sorting condition."

Lemma A.2 (Sorting condition). Let $\left(c^{M}, c^{A}\right)$ and $\left(\tilde{c}^{M}, \tilde{c}^{A}\right)$ denote different Donor contribution choices with $p\left(c^{M}, c^{A}\right)>$ $p\left(\tilde{c}^{M}, \tilde{c}^{A}\right)$. In any equilibrium, if the bad type of Donor weakly prefers to give $\left(c^{M}, c^{A}\right)$ over $\left(\tilde{c}^{M}, \tilde{c}^{A}\right)$ then the good type of Donor strictly prefers to give $\left(c^{M}, c^{A}\right)$ over $\left(\tilde{c}^{M}, \tilde{c}^{A}\right)$.

Proof of Lemma A.2. This follows from Lemma 2 and the analysis in the text of the paper.

\section{A.3 Proofs of main results}

\section{A.3.1 Existence result in baseline model}

Proposition 1. There is a separating equilibrium if and only if

$$
\frac{\mathbb{E}\left[x_{M}^{\text {Sep }} \mid \hat{s}_{D}=G, s_{D}=B\right]}{\mathbb{E}\left[x_{M}^{\text {Sep }} \mid \hat{s}_{D}=B, s_{D}=B\right]} \geq \frac{p(0,1)(b-1)+1}{p(1,0)(b-1)+1} .
$$

In a separating equilibrium the bad type of Donor contributes its entire budget to the Ally and the good type of Donor's contribution increases the probability of electing the Moderate enough to deter imitation by bad types.

Proof of Proposition 1. Suppose $\frac{\mathbb{E}\left[x_{M}^{S e p} \mid \hat{s}_{D}=G, s_{D}=B\right]}{\mathbb{E}\left[x_{M}^{S e} \mid \hat{s}_{D}=B, s_{D}=B\right]} \geq \frac{p(0,1)(b-1)+1}{p(1,0)(b-1)+1}$. In any separating equilibrium the bad type makes the contribution that maximizes its electoral benefit, which is to contribute $\left(c^{M}, c^{A}\right)=(0,1)$. Define a function $\Delta\left(c^{M}, c^{A}\right)=$ $\frac{\mathbb{E}\left[x_{M}^{S e p} \mid \hat{s}_{D}=G, s_{D}=B\right]}{\mathbb{E}\left[x_{M}^{S e p} \mid \hat{s}_{D}=B, s_{D}=B\right]}-\frac{p(0,1)(b-1)+1}{p\left(c^{M}, c^{A}\right)(b-1)+1}$. When $\Delta\left(c^{M}, c^{A}\right)<0$ the bad type would send $\left(c^{M}, c^{A}\right)$ rather than $(0,1)$ in order to imitate the good type and when $\Delta\left(c^{M}, c^{A}\right)>0$ she would not. A contribution $\left(c^{M}, c^{A}\right)$ for which $\Delta\left(c^{M}, c^{A}\right)=0$ 
is one that makes the bad type indifferent between contributing $(0,1)$ and imitating the good type. By continuity of $p, \Delta$ is continuous in $\left(c^{M}, c^{A}\right)$. Since $\frac{\mathbb{E}\left[x_{M}^{S e p} \mid \hat{s}_{D}=G, s_{D}=B\right]}{\mathbb{E}\left[x_{M}^{S e p} \mid \hat{s}_{D}=B, s_{D}=B\right]}<1$ we have $\Delta(0,1)=\frac{\mathbb{E}\left[X_{M}^{S e p} \mid \hat{s}_{D}=B, s_{D}=B\right]}{\mathbb{E}\left[x_{M}^{S e p} \mid \hat{s}_{D}=G, s_{D}=B\right]}-1<0$. Since $\frac{\mathbb{E}\left[x_{M}^{\text {Sep }} \mid \hat{s}_{D}=G, s_{D}=B\right]}{\mathbb{E}\left[x_{M}^{\text {Sep }} \mid \hat{s}_{D}=B, s_{D}=B\right]} \geq \frac{p(0,1)(b-1)+1}{p(1,0)(b-1)+1}$ we also have $\Delta(0,1) \geq 0$. By the intermediate value theorem there must be a contribution $\left(c_{G}^{M *}, c_{G}^{A *}\right)$ such that $\Delta\left(c_{G}^{M *}, c_{G}^{A *}\right)=0$. Since the good type strictly prefers to give this $\left(c_{G}^{M *}, c_{G}^{A *}\right)$ over $(0,1)$ by Lemma A.2, there is a separating equilibrium in which the good type sends $\left(c_{G}^{M *}, c_{G}^{A *}\right)$ and the bad type sends $(0,1)$

\section{A.3.2 Costly contributions}

The following Lemma shows that the Donor sorting in Lemma A.2 also holds in the model with costly contributions. This result is essential, as it was for proving Proposition 1, for the proof of Proposition 3, which shows we can still support a separating equilibrium in a model with costly contributions.

Our first step is to characterize the contribution that would be made by the bad type of Donor in a separating equilibrium. In such an equilibrium, the bad type would take as given that politicians would learn its type and maximize $-\mathbb{E}\left[x_{M}^{S e p} \mid \hat{s}_{D}=B, s_{D}=B\right] \frac{p\left(0, c^{A}\right)(b-1)+1}{b}-k c^{A}$ subject to $0 \leq c^{A} \leq 1$. Note that $c^{M}$ is set equal to zero because the optimal choice for the bad type in a separating equilibrium cannot involve contributions to the Moderate. Given the properties of the beta-binomial model and the fact that $\mathbb{E}\left[x_{M}^{S e p} \mid \hat{s}_{D}=B, s_{D}=B\right]=\mathbb{E}\left[\theta \mid s_{D}=B\right]$ (by the law of total expectations), we can set $\mathbb{E}\left[x_{M}^{S e p} \mid \hat{s}_{D}=B, s_{D}=B\right]=\frac{\alpha+1}{\alpha+\beta+1}$. Thus, the bad type's contribution maximizes

$$
\frac{\alpha+1}{\alpha+\beta+1} \frac{p\left(0, c^{A}\right)(b-1)+1}{b}-k c^{A}
$$

subject to $0 \leq c^{A} \leq 1$. An interior solution satisfies the first-order condition ${ }^{1}$

$$
-\frac{\partial p\left(0, c^{A}\right)}{\partial c^{A}}=k \frac{b(\alpha+\beta+1)}{(b-1)(\alpha+1)}
$$

Naturally, the interior solution is decreasing in contribution costs $k$ and increasing in the Ally's bias $b$.

When contribution costs are sufficiently small $\left(k \leq-\frac{\partial p\left(c^{M}, c^{A}\right)}{\partial c^{A}} \frac{(\alpha+1)(b-1)}{b(\alpha+\beta+1)}\right)$ there is no interior solution and in a separating equilibrium the bad type of $D$ would contribute its entire budget toward electing the Ally. In this case, the main result is identical to the one in the baseline model. To see why, recall that Proposition 1 relied on a determination of whether the bad type of Donor would go from giving its whole budget to the Ally to giving its whole budget to the Moderate in order to persuade the politicians and then appealing to continuity to show that some contribution makes the bad type indifferent between separating and imitating. If costs are small enough that the bad type would give its whole budget then we can make the same argument in this case: the comparison is between two contributions with

\footnotetext{
${ }^{1}$ The second order condition is $-\frac{b-1}{b} \frac{\alpha+1}{\alpha+\beta+1} \frac{\partial^{2} p\left(0, c^{A}\right)}{\partial c^{A^{2}}}<0$

which is satisfied when $\frac{\partial^{2} p\left(0, c^{A}\right)}{\partial c^{A^{2}}}>0$.
} 
identical direct costs and therefore the bad type's preference depends only on the electoral and persuasion effects as before. The first result establishes that the sorting condition still holds in the model with contribution costs if and only if if $b>1$ and electoral effects of contributions are not zero.

Lemma A.3. Lemma A.2 holds in the costly contributions model if and only if $b>1$ and $p$ is not constant.

Proof of Lemma A.3. We begin by restating Lemma A.2: Let $\left(c^{M}, c^{A}\right)$ and $\left(\tilde{c}^{M}, \tilde{c}^{A}\right)$ denote different contribution choices with $p\left(c^{M}, c^{A}\right)>p\left(\tilde{c}^{M}, \tilde{c}^{A}\right)$. In any equilibrium if the bad type of $D$ weakly prefers to give $\left(c^{M}, c^{A}\right)$ over $\left(\tilde{c}^{M}, \tilde{c}^{A}\right)$ then the good type of $D$ strictly prefers to give $\left(c^{M}, c^{A}\right)$ over $\left(\tilde{c}^{M}, \tilde{c}^{A}\right)$.

To prove that this also holds in the costly contributions model, note as before that if the bad type is indifferent in equilibrium between $\left(c^{M}, c^{A}\right)$ and $\left(\tilde{c}^{M}, \tilde{c}^{A}\right)$ and $p\left(c^{M}, c^{A}\right)>p\left(\tilde{c}^{M}, \tilde{c}^{A}\right)$ then it must be the case the $\hat{s}\left(c^{M}, c^{A}\right)=0$ and $\hat{s}\left(\tilde{c}^{M}, \tilde{c}^{A}\right)=1$. For any type $s_{D} \in\{G, B\},\left(c^{M}, c^{A}\right)$ is weakly preferred to $\left(\tilde{c}^{M}, \tilde{c}^{A}\right)$ if

$$
\left.\mathbb{E}\left[x_{M}^{S e p} \mid \hat{s}_{D}=B, s_{D}\right] \frac{p\left(\tilde{c}^{M}, \tilde{c}^{A}\right)(b-1)+1}{b}-\hat{s}_{D}=G, s_{D}\right] \frac{p\left(c^{M}, c^{A}\right)(b-1)+1}{b} \geq k\left(c^{M}+c^{A}\right)-k\left(\tilde{c}^{M}+\tilde{c}^{A}\right) .
$$

The right-hand-side of equation 5 does not depend on $G$ 's type so it is sufficient to prove that

$$
\begin{array}{r}
\mathbb{E}\left[x_{M}^{S e p} \mid \hat{s}_{D}=B, G\right] \frac{p\left(\tilde{c}^{M}, \tilde{c}^{A}\right)(b-1)+1}{b}-\mathbb{E}\left[x_{M}^{S e p} \mid \hat{s}_{D}=G, s_{D}=G\right] \frac{p\left(c^{M}, c^{A}\right)(b-1)+1}{b}> \\
\mathbb{E}\left[x_{M}^{S e p} \mid \hat{s}_{D}=B, s_{D}=B\right] \frac{p\left(\tilde{c}^{M}, \tilde{c}^{A}\right)(b-1)+1}{b}-\mathbb{E}\left[x_{M}^{S e p} \mid \hat{s}_{D}=G, s_{D}=B\right] \frac{p\left(c^{M}, c^{A}\right)(b-1)+1}{b}
\end{array}
$$

which implies that if inequality (5) holds weakly for the bad type then it holds strictly for the good type. Re-arranging inequality (6) yields

$$
\frac{\mathbb{E}\left[x_{M}^{S e p} \mid \hat{s}_{D}=B, s_{D}=G\right]-\mathbb{E}\left[x_{M}^{S e p} \mid \hat{s}_{D}=B, s_{D}=B\right]}{\mathbb{E}\left[x_{M}^{S e p} \mid \hat{s}_{D}=G, s_{D}=G\right]-\mathbb{E}\left[x_{M}^{S e p} \mid \hat{s}_{D}=G, s_{D}=B\right]} \leq \frac{p\left(\tilde{c}^{M}, \tilde{c}^{A}\right)(b-1)+1}{p\left(\tilde{c}^{M}, \tilde{c}^{A}\right)(b-1)+1} .
$$

Since $p\left(c^{M}, c^{A}\right)>p\left(\tilde{c}^{M}, \tilde{c}^{A}\right)$ we have $\frac{p\left(\tilde{c}^{M}, \tilde{c}^{A}\right)(b-1)+1}{p\left(\tilde{c}^{M}, \tilde{c}^{A}\right)(b-1)+1}>1$. Using the beta-binomial properties and substituting the appropriate expectations into equation (5) yields

$$
\begin{aligned}
& \mathbb{E}\left[x_{M}^{S e p} \mid \hat{s}_{D}=B, s_{D}=G\right]=\frac{\alpha}{\alpha+\beta+1} \frac{\alpha+2}{\alpha+\beta+2}+\left(1-\frac{\alpha}{\alpha+\beta+1}\right) \frac{\alpha+1}{\alpha+\beta+2}=\frac{\alpha}{\alpha+\beta+1}+\frac{1}{\alpha+\beta+2} \\
& \mathbb{E}\left[x_{M}^{S e p} \mid \hat{s}_{D}=B, s_{D}=B\right)=\frac{\alpha+1}{\alpha+\beta+1} \frac{\alpha+2}{\alpha+\beta+2}+\left(1-\frac{\alpha+1}{\alpha+\beta+1}\right) \frac{\alpha+1}{\alpha+\beta+2}=\frac{\alpha+1}{\alpha+\beta+1} \\
& \mathbb{E}\left[x_{M}^{S e p} \mid \hat{s}_{D}=G, s_{D}=G\right]=\frac{\alpha}{\alpha+\beta+1} \frac{\alpha+1}{\alpha+\beta+2}+\left(1-\frac{\alpha}{\alpha+\beta+1}\right) \frac{\alpha}{\alpha+\beta+2}=\frac{\alpha}{\alpha+\beta+1} \\
& \mathbb{E}\left[x_{M}^{S e p} \mid \hat{s}_{D}=G, s_{D}=B\right]=\frac{\alpha+1}{\alpha+\beta+1} \frac{\alpha+1}{\alpha+\beta+2}+\left(1-\frac{\alpha+1}{\alpha+\beta+1}\right) \frac{\alpha}{\alpha+\beta+2}=\frac{\alpha+1}{\alpha+\beta+1}-\frac{1}{\alpha+\beta+2} .
\end{aligned}
$$


Therefore

$$
\frac{\mathbb{E}\left[x_{M}^{S e p} \mid \hat{s}_{D}=B, s_{D}=G\right]-\mathbb{E}\left[x_{M}^{S e p} \mid \hat{s}_{D}=B, s_{D}=B\right)}{\mathbb{E}\left[x_{M}^{S e p} \mid \hat{s}_{D}=G, s_{D}=G\right]-\mathbb{E}\left[x_{M}^{S e p} \mid \hat{s}_{D}=G, s_{D}=B\right]}=\frac{1 / \alpha+\beta+2}{1 / \alpha+\beta+2}=1,
$$

which implies inequality (7). This shows that inequality (5) holds strictly for the good type if it holds weakly for the bad type, which proves the lemma. Note that, if $b=1$ or electoral costs are non-existent, both ratios equal one so the strict inequality cannot hold.

Proposition 3. Let $c^{*}$ be the optimal contribution to the Ally for the bad type of Donor in a separating equilibrium to the costly contribution game. If

$$
\mathbb{E}\left[x_{M}^{S e p} \mid \hat{s}_{D}=B, s_{D}=B\right] \frac{p\left(0, c^{*}\right)(b-1)+1}{b}-\mathbb{E}\left[x_{M}^{S e p} \mid \hat{s}_{D}=G, s_{D}=B\right] \frac{p(1,0)(b-1)+1}{b} \leq k\left(c^{M}+c^{A}-c^{*}\right)
$$

then there exists a separating equilibrium in which the bad type of Donor contributes $\left(0, c^{*}\right)$ and the good type of Donor contributes some $\left(\tilde{c}^{M}, \tilde{c}^{A}\right)$ with $p\left(\tilde{c}^{M}, \tilde{c}^{A}\right)>p\left(0, c^{*}\right)$. There is no separating equilibrium if $b$ is too close to one or if the electoral effects of contributions are too small.

Proof of Proposition 3. The proof is nearly identical to the proof of Proposition 1. Suppose

$$
\mathbb{E}\left[x_{M}^{S e p} \mid \hat{s}_{D}=B, s_{D}=B\right] \frac{p\left(0, c^{*}\right)(b-1)+1}{b}-\mathbb{E}\left[x_{M}^{S e p} \mid \hat{s}_{D}=G, s_{D}=B\right] \frac{p(1,0)(b-1)+1}{b} \leq k\left(1-c^{*}\right) .
$$

Recall that in any separating equilibrium the bad type contributes $\left(0, c^{*}\right)$. Define a function $\Delta^{*}\left(c^{M}, c^{A}\right)=\mathbb{E}\left[x_{M}^{S e p} \mid \hat{S}_{D}=\right.$ $\left.B, s_{D}=B\right] \frac{p\left(0, c^{*}\right)(b-1)+1}{b}-\mathbb{E}\left[x_{M}^{S e p} \mid \hat{s}_{D}=G, s_{D}=B\right] \frac{\left.p\left(c^{M}, c^{A}\right)\right)(b-1)+1}{b} k\left(c^{M}+c^{A}-c^{*}\right)$ as the increase in expected utility from deviating to $\left(c^{M}, c^{A}\right)$ assuming this deviation induces the belief $\hat{s}\left(c^{M}, c^{A}\right)=0$. The point at which $\Delta^{*}\left(c^{M}, c^{A}\right)=0$ is the point at which the bad type is indifferent, positive values indicate that the bad type of $D$ would strictly prefer to deviate, and negative values indicate that the bad type of $D$ would not deviate. $\Delta^{*}\left(c^{M}, c^{A}\right)$ is continuous in $\left(c^{M}, c^{A}\right)$ and in $c^{M}-c^{A}$, both by continuity of $p$. The assumption (inequality (8)) gives us $\Delta^{*}(1,0)<0$. Clearly $\Delta^{*}\left(0, c^{*}\right)>0$. Thus, by the intermediate value theorem and by continuity of $\Delta^{*}$ with respect to $c^{M}-c^{A}$, there must be some contribution $\left(\tilde{c}^{M}, \tilde{c}^{A}\right)$ such that $\Delta^{*}\left(\tilde{c}^{M}, \tilde{c}^{A}\right)=0$, meaning that bad type is indifferent between contributing $\left(0, c^{*}\right)$ and imitating the good type by giving this contribution. by Lemma A.3 this constitutes a separating equilibrium of the game. Furthermore, if Inequality (8) holds then there can be no contribution that would deter the bad type from imitating, which means any equilibrium is pooling. 


\section{B Extensions and robustness}

\section{B.1 Comparing electoral and policy effects}

Separating equilibrium in the baseline model requires that

$$
\frac{p(1,0)(b-1)+1}{p(0,1)(b-1)+1} \geq \frac{\mathbb{E}\left[x_{M}^{S e p} \mid \hat{s}_{D}=B, s_{D}=B\right)}{\mathbb{E}\left[x_{M}^{S e p}\left(\hat{s}_{D}=G, s_{D}=B\right]\right.} .
$$

This is a comparison between the maximum electoral effect of contributions and the policy effect of the Donor posing as a good type when he is a bad type. In this section we provide some numerical calculations to show that the size of the required electoral effect varies considerably with the precision of the prior and that in many cases the electoral effect can be quite small.

For these calculations, we maintain the following assumptions:

1. Prior beliefs follow a Beta distribution with an expectation of $\frac{2}{3}$. This holds when the parameters of the Beta distribution satisfy $\alpha=2 \beta$. Given this assumption, the variance of the prior distribution is decreasing in $\beta$. We maintain this assumption so that we may vary the prior distribution without conflating increased precision with changes in the expectation.

2. The election is a coin flip when all contributions go to the Ally and the probability the Moderate wins increases by $\delta>0$ when all contributions go to the Moderate. Thus, $\delta$ is the change in the probability that the Moderate wins due to the maximum change in the Donor's contributions. This corresponds to a slight ex ante advantage for the Moderate, since the Moderate must be more likely to win if both contributions are zero.

With these two assumptions in hand, our condition for separating equilibrium becomes

$$
\frac{(.5+\delta)(b-1)+1}{.5(b-1)+1} \geq \frac{(2 \beta+1)(3 \beta+2)}{6 \beta^{2}+4 \beta+1} .
$$

The right-hand side of (9) is found by using the expression for $\mathbb{E}\left[x_{M}^{\operatorname{Sep}} \mid \hat{s}_{D}=B, s_{D}=B\right]$ and $\mathbb{E}\left[x_{M}^{S e p} \mid \hat{s}_{D}=G, s_{D}=B\right]$ for the Beta distribution from the proof of Lemma A.3 in the main text, substituting $\alpha=2 \beta$, and simplifying.

To illustrate the implications of Inequality (9) for the size of electoral effects needed to support separating equilibrium we consider a collection of Beta priors satisfying our assumptions and compute, for several different polarization values $b$, the minimum value of $\delta$ that would be required to support a separating equilibrium. The densities of the prior distributions used in our calculations are provided in Figure 1. Notably, as the value of $\beta$ increases so does the precision of beliefs. However, none of the prior beliefs are particularly extreme: even the most precise prior belief in our example has an interquartile range of over .01 units on either side of the expectation. Though it is hard to 


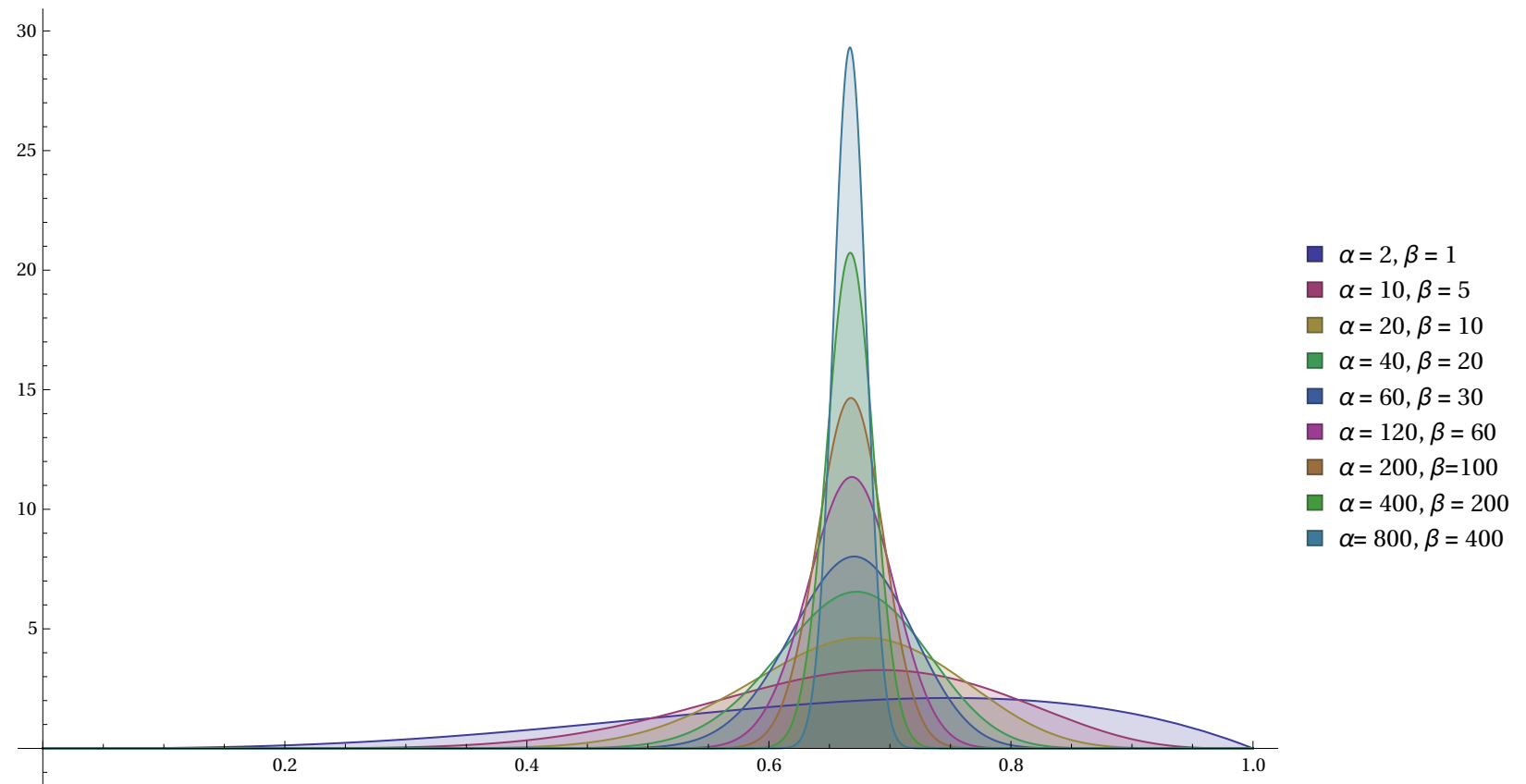

Figure 1: Densities of Beta priors used in calculations

know which prior beliefs are empirically justifiable, we think of the more precise priors as more appropriate for fairly well-established policy areas for which groups are likely advocating for minor changes to policy relative to the range of possible regulatory regimes. In fact, one way we can think of the priors is to imagine that all players began with uniform priors and received a number of public signals before the start of the game. In this case, the number of public signals is $\alpha+\beta-2$. For more salient policies the level of existing information is likely to be larger.

For each of these priors, we compute the right-hand side of (9). We then solve for the minimum level of $\delta$ that can support a separating equilibrium when $b$ is equal to $2,4,6,8$, and 10 . The chosen values of $b$ are meant to represent a wide range of possible polarization values. The results are showin in Figure 2. Each entry in the table represents the minimum $\delta$ at which there is a separating equilibrium for these parameter values.

One message from Figure 2 is that the meaning of having a "large enough" electoral effect to generate informative contributions varies considerably according to the prior beliefs and polarization levels. For the least precise prior beliefs and the lowest polarization level, contributions would need to have an implausibly large effect on elections to generate our separating equilibrium. However, with high polarization and high prior precision, the required effect is quite small. In the most extreme case, a change from the maximum contribution to the Ally to a maximum contribution to the Moderate must only change the probability that the Ally is elected from .5 to .5008 .

It is admittedly difficult using existing estimates to place clear bounds on what constitutes a reasonable value of $\delta$ in the context of a model like this one. Though there is a sizable body of empirical work on the electoral effects of campaign spending, most of the estimates are of aggregate effects of policies rather than of the marginal effect of 


\begin{tabular}{|l|l|l|l|l|l|}
\hline$b$ & 2 & 4 & 6 & 8 & 10 \\
\hline$\alpha=2, \beta=1$ & 0.3180 & 0.1770 & 0.1480 & 0.1360 & 0.1300 \\
\hline$\alpha=10, \beta=5$ & 0.1400 & 0.0780 & 0.0655 & 0.0602 & 0.0572 \\
\hline$\alpha=20, \beta=10$ & 0.0725 & 0.0403 & 0.0339 & 0.0311 & 0.0296 \\
\hline$\alpha=40, \beta=20$ & 0.0369 & 0.0205 & 0.0172 & 0.0158 & 0.0150 \\
\hline$\alpha=60, \beta=30$ & 0.0247 & 0.0137 & 0.0115 & 0.0106 & 0.0101 \\
\hline$\alpha=120, \beta=60$ & 0.0124 & 0.0069 & 0.0058 & 0.0053 & 0.0051 \\
\hline$\alpha=200, \beta=100$ & 0.0075 & 0.0042 & 0.0035 & 0.0032 & 0.0030 \\
\hline$\alpha=400, \beta=200$ & 0.0037 & 0.0021 & 0.0017 & 0.0016 & 0.0015 \\
\hline$\alpha=800, \beta=400$ & 0.0019 & 0.0010 & 0.0009 & 0.0008 & 0.0008 \\
\hline
\end{tabular}

Figure 2: For each prior and level of polarization, cell entry is the minimum level of $\delta$ to support a separating equilibrium.

particular contributions amounts and most use vote or seat shares as outcomes rather than particular electoral outcomes. One estimate was that the decision in Citizens United caused about a 4 percentage point increase in Republican state legislator's probabilities of reelection on average, and around 10 percentage points in some states, comparing states who previously disallowed these expenditures to those that did not using a difference-in-differences design (Klumpp, Mialon and Williams, 2016). Importantly, these are averages over many elections, many of which are likely to be in safe districts, so the effects on certain individual incumbents are likely considerably larger than this. Furthermore, as Klumpp, Mialon and Williams (2016) noted, in many states a small number of individuals or organizations accounted for a large percentage of the independent expenditures in one state. For instance, $72 \%$ of independent expenditures in North Carolina were from groups associated with one man named Art Pope in 2010. Given this large effect and the concentration of outside spending among a small group of donors, it seems to us that many of the effect sizes in Figure 2 are reasonably small relative to the likely effects of contributions for some elections and donors.

\section{B.1.1 Multiple donors}

Example B.1. Consider an $n$-donor example of the model. Suppose $\theta$ is uniform on $[0,1]$ and let $b=10$. Now suppose there are $n$ identical donors that each receive conditionally independent signals and simultaneously choose contribution levels. Letting $T_{M}$ and $T_{A}$ denote the total amounts of contributions to the Moderate and the Ally, respectively. The probability that the Moderate is elected is then,

$$
p\left(T_{M}, T_{A}, n\right)=\log \left(\frac{1+e}{2}+\frac{e-1}{2 n}\left(T_{M}-T_{A}\right)\right)
$$


where the letter $e$ here represents the exponential constant and the log has base e. $p()$ is therefore log-linear and the intercept and slope are chosen to ensure that all probabilities fall between zero and one. We will show that there is a symmetric separating equilibrium even for large $n$. A separating equilibrium profile to this game involves each donor choosing $\left(c^{M}, c^{A}\right)=(0,1)$ when he is a bad type and $\left(c^{M}, c^{A}\right)=\left(c^{M *}, 0\right)$ for some $c^{M *}>0$ when he is a good type. The winning candidate, in turn, updates her beliefs assuming all contributions with $c^{M}>c^{M *}$ and $c^{A}=0$ indicate signals of $G$ and any contribution with $c^{A} \geq 0$ or $c^{M}<c^{M *}$ indicate signals of B. Since the uniform is a $\operatorname{Beta}(1,1)$ distribution, this means that the posterior distribution of $\theta$ for candidate $j$ following a set of $n$ contributions and her own signal, letting $S$ denote the candidate's belief about the total number of bad signals, is distributed $\operatorname{Beta}\left(1+S+\mathbb{I}_{\left(s_{j}=B\right)}, 1+n-\right.$ $\left.S-\mathbb{I}_{\left(s_{j}=B\right)}\right)$, where $\mathbb{I}_{\left(s_{j=B)}\right.}$ is an indicator that takes the value of one when $s_{j}=B$ and zero when $s_{j}=G$, and policy is chosen accordingly (equal to the expectation of $\theta$ for Moderate candidates and $1 / b$ times that expectation for Allies). Using this information, we can compute each type of donor's expected utility for choosing each signal on the path of play. The expected utility for type $s_{D}$ of contributing $\left(c^{M *}, 0\right)$ is given by,

$$
U_{c^{M *}}\left(s_{D}, c^{M *}, n\right)=-\mathbb{E}\left[\frac{(S+1)\left(1-p\left(c^{M}(n-S)+c^{M}, S, n\right)\right)}{b(n+2+1)}+\frac{(S+1) p\left(c^{M}(n-S)+c^{M}, S, n\right)}{n+2+1}\right],
$$

where the expectation is taken with respect to $S$, which is distributed Beta-Binomial with parameters $\alpha=1+\mathbb{I}_{\left(s_{D}=B\right)}$, $\beta=2-\mathbb{I}_{\left(s_{D}=B\right)}$ and $n=n$ (the first two parameters are the updated beliefs about $\theta$ from the signal $s_{D}$ and the sample size $n$ reflects the number of signals - all other donors plus one candidate). Similarly, the expected utility from contributing $(0,1)$ is,

$$
U_{0}\left(s_{D}, c^{M *}, n\right)-\mathbb{E}\left[\frac{(S+1+1)\left(1-p\left(c^{M}(n-S), S+1, n\right)\right)}{b(n+2+1)}+\frac{(S+1+1) p\left(c^{M}(n-S), S+1, n\right)}{n+2+1}\right],
$$

where $S$ is distributed in the same way as above. For this example we compute these expectations numerically and omit the analytical details of the calculations.

Notice that, unlike in the single donor model, the expected policy and the probability of electing the Moderate for a given contribution both depend on the players' types since they also affect expectations over other players' contributions. Nevertheless, our sorting condition still holds. The next plot verifies that the good type of donor has a stronger incentive to choose $\left(c^{M *}, 0\right)$ over $(0,1)$ than does the bad type of donor. This holds if the following expression, $\delta\left(c^{M *}, n\right)$ which is the difference in differences in expected utility for contributing $c^{M *}$ given $n$ donors, is negative for a given $n$ and all $c^{M *} \in(0,1)$ :

$$
\delta\left(c^{M *}, n\right)=\left(U_{c^{M *}}\left(1, c^{M *}, n\right)-U_{0}\left(1, c^{M *}, n\right)\right)-\left(U_{c^{M *}}\left(1, c^{M *}, n\right)-U_{0}\left(1, c^{M *}, n\right)\right) .
$$

Figure 3 verifies that this holds for several values of $n$. 


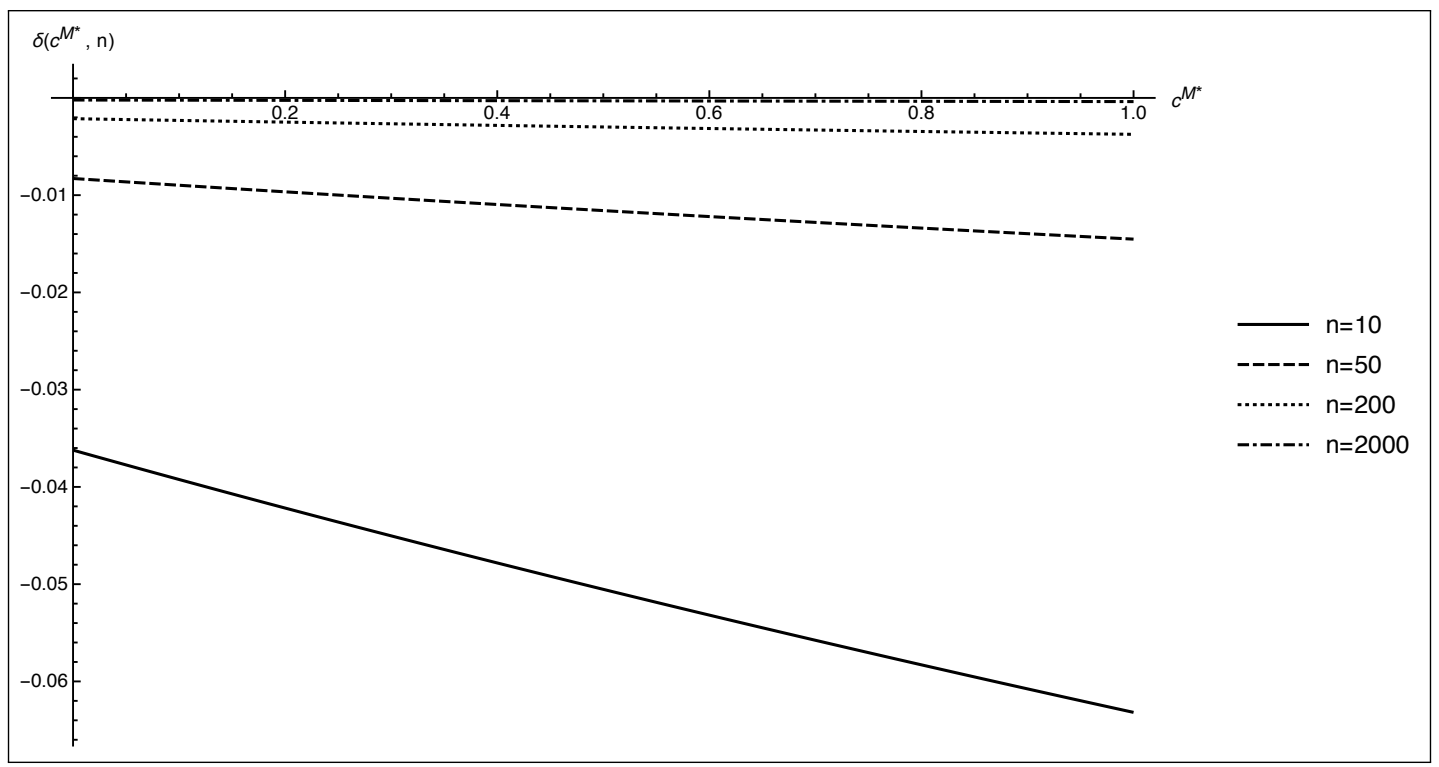

Figure 3: The lines show values of $\delta\left(c^{M *}, n\right)$ for each possible value of $c^{M *}$ and various values of $n$. Values below 0 verify our sorting condition: bad types of the donor have less of an incentive to contribute to the Moderate in order to be viewed as a good type of donor. Note also that the lines are decreasing in $c^{M *}$, since naturally this difference in incentives widens as we increase the required contribution and therefore increase the electoral disincentive to do so. However, the slope of the lines are lower for higher values of $n$ since the importance of any one contribution diminishes as $n$ increases.

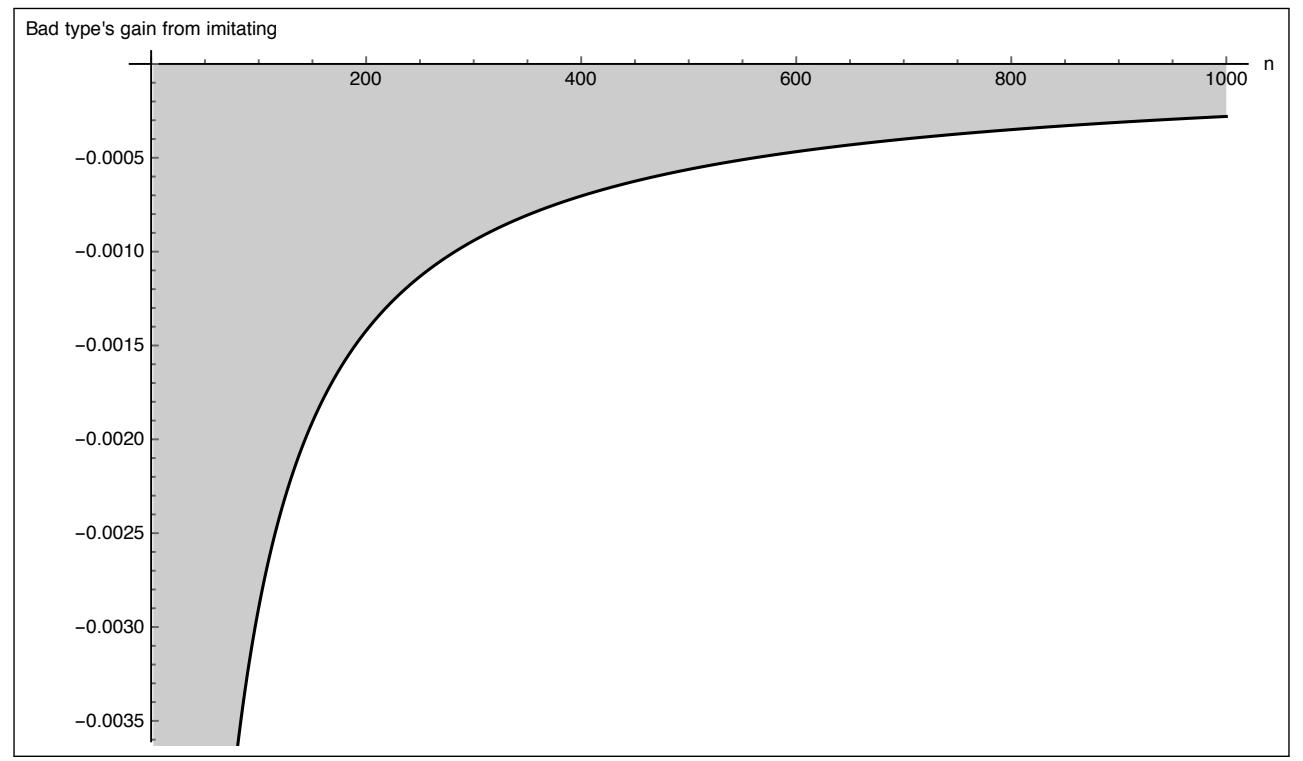

Figure 4: This plot shows the bad type of donor's net utility from deviating from the separating equilibrium by giving the maximum amount to the Moderate, for each value of $n$. Negative values indicate that the bad type would not deviate, which means that we can support a symmetric separating equilibrium for that value of $n$.

The sorting condition implies that if there is a contribution to the moderate $c^{M *}>0$ that would make the bad type of donor indifferent between sending $\left(c^{M *}, 0\right)$ and $(0,1)$ given the proposed strategy profile, the good type would strictly benefit from separating and sending $\left(c^{M *}, 0\right)$. We must now verify that some contribution would make the bad type of donor indifferent. As in the one donor model, we do so by showing that the bad type would not send 
the maximum amount to the Moderate. Then, by appealing to continuity, we know that some contribution makes the bad type indifferent. Figure 4 shows that this is true even for very large numbers of donors. In this figure, the number of donors is on the $x$-axis and the $y$-axis represents the bad type's utility gain from imitating by sending $(1,0)$. We note that this utility gain is negative for all of the examined values of $n$, which shows that the bad type can be deterred from imitating and therefore there is a symmetric separating equilibrium. Note that this example does not show that separating equilibria are always robust to increasing the number of donors. In fact, for smaller values of $b$ the separating equilibrium is only sustainable for relatively small numbers of donors. As we have noted, the answer depends on how quickly the electoral and persuasion components of the effects of contributions go to zero as $n$ gets large: if the electoral effects converge to zero more slowly, as they do in this example, then the separating equilibrium is sustained for a larger number of donors (i.e., higher values of $n$ ).

\section{B.2 Separating conditions for more general Ally utility function}

In this section we consider a more general candidate utility function in which the Ally might have an additive bias (as in Crawford-Sobel and other works) as well as the multiplicative bias that is in the baseline model. The purpose of this extension is to illustrate the impact of our assumption that candidate preferences diverge from each other more as their expectations about $\theta$ increase, which is the third assumption regarding candidate preferences discussed in the main text. The general message is that these two types of candidate preferences work much differently and the style of preferences used in the model are key to the mechanism in the paper. The key difference is that, with the multiplicative candidate biases in our model, the bad type of Donor perceives greater electoral stakes than does the good type and is therefore less willing to help the Moderate in order to influence the politicians. With Crawford-Sobelstyle preferences, the two candidates' policy choices as a function of their expectations about $\theta$ are two parallel lines, so the perceived electoral stakes do not change as expectations about $\theta$ change. Below, however, we combine the two types of preferences. If Ally candidates have some additive bias in addition to the multiplicative bias, the conditions to support a separating equilibrium can be easier to satisfy, since the constant differences between the candidates serve as additional deterrent to the bad type. However, this only works because the sorting condition distinguishing between the types is bolstered by the mechanism from the baseline model. In this way, additive differences between candidates operate in much the same way as contribution costs.

Consider a variant of our original game with the following preferences for the ally:

$$
u_{A}(\theta, x)=-(\theta b-x+\gamma)^{2}
$$

with $\gamma \geq 0$ and $b>1$. The $\gamma$ parameter is the bias as in Crawford and Sobel and $b$ is the bias from our original model. Our purpose is to illustrate why the parameter $b$ is essential to the mechanism driving separating equilibria in this 
game while $\gamma$ is not.

Given a belief about $\theta$ the Ally's best reply then is to set $x=\mathbb{E}[\theta] / b-\gamma$. A player of type $s_{D}$ now prefers $\left(c^{M}, c^{A}\right)$ over $\left(\tilde{c}^{M}, \tilde{c}^{A}\right)$ if,

$$
\begin{aligned}
& -p\left(c^{M}, c^{A}\right) \mathbb{E}\left[x_{M}^{S e p} \mid \hat{s}_{D}\left(c^{M}, c^{A}\right), s_{D}\right]-\left(1-p\left(c^{M}, c^{A}\right)\right)\left(\frac{\mathbb{E}\left[x_{M}^{S e p} \mid \hat{s}_{D}\left(c^{M}, c^{A}\right), s_{D}\right]}{b}-\gamma\right) \geq \\
& -p\left(\tilde{c}^{M}, \tilde{c}^{A}\right) \mathbb{E}\left[x_{M}^{S e p} \mid \hat{s}_{D}\left(\tilde{c}^{M}, \tilde{c}^{A}\right), s_{D}\right]-\left(1-p\left(\tilde{c}^{M}, \tilde{c}^{A}\right)\right)\left(\frac{\left(\mathbb{E}\left[x_{M}^{S e p} \mid \hat{s}_{D}\left(\tilde{c}^{M}, \tilde{c}^{A}\right), s_{D}\right]\right.}{b}-\gamma\right)
\end{aligned}
$$

Rearranging, type $s_{D}$ would prefer $\left(c^{M}, c^{A}\right)$ over $\left(\tilde{c}^{M}, \tilde{c}^{A}\right)$ if,

$$
\begin{array}{r}
\mathbb{E}\left[x_{M}^{S e p} \mid \hat{s}_{D}\left(c^{M}, c^{A}\right), s_{D}\right]\left(\frac{p\left(c^{M}, c^{A}\right)-1}{b}-p\left(c^{M}, c^{A}\right)\right)-\mathbb{E}\left[x_{M}^{S e p} \mid \hat{s}_{D}\left(\tilde{c}^{M}, \tilde{c}^{A}\right), s_{D}\right] \\
\left(\frac{p\left(\tilde{c}^{M}, \tilde{c}^{A}\right)-1}{b}-p\left(\tilde{c}^{M}, \tilde{c}^{A}\right)\right) \\
\geq \gamma\left(p\left(c^{M}, c^{A}\right)-p\left(\tilde{c}^{M}, \tilde{c}^{A}\right)\right) .
\end{array}
$$

Consider a separating profile in which (a) the contribution $\left(\hat{c}^{M}, 0\right)$ induces the belief that $s_{D}=G$, (b) the contribution $(0,1)$ induces the belief that $s_{D}=B$. Type $s_{D}$ prefers $\left(\hat{c}^{M}, 0\right)$ over $(0,1)$ if

$$
\begin{aligned}
\mathbb{E}\left[x_{M}^{S e p} \mid \hat{s}_{D}=G, s_{D}\right]\left(\frac{p\left(\hat{c}^{M}, 0\right)-1}{b}-p\left(\hat{c}^{M}, 0\right)\right)-\mathbb{E}\left[x_{M}^{S e p} \mid \hat{s}_{D}=B, s_{D}\right] & \left(\frac{p(0,1)-1}{b}-p(0,1)\right) \\
& \geq \gamma\left(p\left(\hat{c}^{M}, 0\right)-p(0,1)\right) .
\end{aligned}
$$

To clarify these conditions, consider the limiting cases as $\gamma \rightarrow 0$ (the original case) and as $b \rightarrow 1$ (Crawford-Sobel-style preferences). When $\gamma=0$ the condition in Equation 11 becomes

$$
\begin{aligned}
\mathbb{E}\left[x_{M}^{S e p} \mid \hat{S}_{D}=G, s_{D}\right]\left(\frac{p\left(\hat{c}^{M}, 0\right)-1}{b}-p\left(\hat{c}^{M}, 0\right)\right)- & \mathbb{E}\left[x_{M}^{S e p} \mid \hat{s}_{D}=B, s_{D}\right]\left(\frac{p(0,1)-1}{b}-p(0,1)\right) \geq 0 \\
\mathbb{E}\left[x_{M}^{S e p} \mid \hat{s}_{D}=G, s_{D}\right]\left(\frac{p\left(\hat{c}^{M}, 0\right)-1}{b}-p\left(\hat{c}^{M}, 0\right)\right) \geq & \mathbb{E}\left[x_{M}^{S e p} \mid \hat{s}_{D}=B, s_{D}\right]\left(\frac{p(0,1)-1}{b}-p(0,1)\right) \\
& \frac{\mathbb{E}\left[x_{M}^{S e p} \mid \hat{s}_{D}=G, s_{D}\right]}{\mathbb{E}\left[x_{M}^{S e p} \mid \hat{s}_{D}=B, s_{D}\right]} \leq \frac{(b-1) p(0,1)+1}{(b-1) p\left(\hat{c}^{M}, 0\right)+1}
\end{aligned}
$$

which is exactly the condition from the baseline model. Note that the inequality changes direction in the last line since the parenthetical terms are negative. When $b \rightarrow 1$ our condition becomes $\mathbb{E}\left[x_{M}^{S e p} \mid \hat{s}_{D}=B, s_{D}\right]-\mathbb{E}\left[x_{M}^{S e p} \mid \hat{s}_{D}=G, s_{D}\right] \geq$ $\gamma\left(p\left(\hat{c}^{M}, 0\right)-p(0,1)\right)$.

We now make a few observations about these conditions. First, while the term $\frac{\mathbb{E}\left[x_{M}^{S e p} \mid \hat{s}_{D}=G, s_{D}\right]}{\mathbb{E}\left[x_{M}^{S e p} \mid \hat{s}_{D}=B, s_{D}\right]}$ is larger when $s_{D}=B$ as shown in Lemma 2, the same cannot be said of the difference $\mathbb{E}\left[x_{M}^{S e p} \mid \hat{s}_{D}=B, s_{D}\right]-\mathbb{E}\left[x_{M}^{S e p} \mid \hat{s}_{D}=G, s_{D}\right]$. For instance, 
if we consider a $\operatorname{Bet} a(\alpha, \beta)$ prior, we would have

$$
\mathbb{E}\left[x_{M}^{S e p} \mid \hat{s}_{D}=B, G\right]-\mathbb{E}\left[x_{M}^{S e p} \mid \hat{s}_{D}=G, G\right]=\mathbb{E}\left[x_{M}^{S e p} \mid \hat{s}_{D}=B, B\right]-\mathbb{E}\left[x_{M}^{S e p} \mid \hat{s}_{D}=G, B\right]=\frac{1}{\alpha+\beta+2}
$$

so that the indifference curves of the good type and bad type would be identical in the model with $b=1$. Second, when $\gamma>0$ and $b>1$ the single crossing condition is still satisfied: the new condition simply adds a type-independent term to the IC condition from the original model. Furthermore Equation (11) implies that increasing $\gamma$ makes separating equilibria easier to obtain as long as we have $b>1$. The reasoning is as follows. Increasing $\gamma$ increases the RHS of Equation (11) meaning that an agent of any type is less inclined to send the contribution $\left(\hat{c}^{M}, 0\right)$. This term increases the electoral stakes involved and makes either type of Donor less inclined to make contributions that increase the likelihood of electing the Moderate. Though this effect is the same for good types as it is for bad types, the main issue for finding separating equilibria is whether or not the bad type can be deterred from imitating. By increasing the costs associated with contributing to the Moderate (or failing to contribute to the Ally), increases in $\gamma$ mean that separating equilibria can be supported more often and at lower overall contribution levels.

\section{Empirical evidence of giving to opponents}

Our model suggests that a certain set of strategic, outcome-oriented donors will sometimes donate to opponents in order to convey some information about their preferred outcomes. Of course, the majority of small donors are not seeking a particular outcome and therefore give money either to express themselves or for pure electoral reasons. We reason that business-oriented donors are relatively less likely to donate for purely expressive reasons and are therefore most likely to be in the category of donors who may strategically give to opponents.

In this section we provide descriptive evidence that (1) donors give to opponents reasonably frequently and (2) giving to opponents is more common among business-oriented donors. Though a complete set of tests of hypotheses derived from the theoretical model is well beyond the scope of this paper, and the existing data do not allow us to rule out competing explanations of the same facts, the descriptive statistics below give some indication that the predicted behavior from our model is plausible and in fact relatively common.

\section{C.1 Data}

The data come from Barber, Canes-Wrone and Thrower (2017), who conducted a survey of donors by sampling from FEC filings. ${ }^{2}$ The sample we use contains 2,903 donors and 63,915 donor-Senator dyads from Senators seeking reelection in 2012. We briefly describe the variables we use in our analysis but refer the reader to the original paper for more detailed information on the sample and dataset. We first present our analysis of donor-Senator dyads. First, we measure whether each respondent is a donor to each particular Senator. We consider respondent $i$ a donor to Senator $j$

\footnotetext{
${ }^{2}$ We used the data from the authors' replication materials in the AJPS Dataverse (Barber, Canes-Wrone and Thrower, 2016).
} 
if she gave more than $\$ 0$ to that Senator's campaign. We dichotomize this variable because the theoretical model does not make a clear prediction about amounts: the contribution to the opponent needs to generate enough electoral cost to make it credible compared to the amount the person could have given to the ally and this number may very well be close to zero considering this opportunity cost.

Second, we need a measure of whether each Senator is an ally or opponent from the perspective of the respondent. To answer this, we use the issue scores generated in Barber, Canes-Wrone and Thrower (2017). Respondents are asked a series of issue questions and these are compared to Senator positions as well as positions taken by the challenger in that election Then a percentage of policy agreement is computed for each respondent relative to the Senator and the challenger. If the respondent agrees with the Senator more often than the challenger, we call them allies. If the respondent agrees with the Senator less often than the challenger, we call them opponents.

Finally, we need a measure of whether the donor belongs to the subgroup in which we expect to find contributions for opponents. We use an interaction of two variables from the dataset: whether the respondent suggested that the candidate's ability to affect his or her "industry or work" was extremely important (Barber, et. al. label these respondents "investors") and whether the Senator's committee matches with the respondents industry or profession. We expect that when both of these things are true the donor may be most motivated to use donations to persuade.

\section{C.2 Dyad-level statistics}

We first analyze descriptive statistics at the dyad level. The probability that any given donor contributes to a particular Senator is low under any condition. However, our purpose is to assess whether contributions to opponents are reasonably likely compared to contributions to allies. In fact, they are. In the condition for which we hypothesize that signaling concerns are most salient, respondents are around $43 \%$ as likely to donate to opponents as to allies. Furthermore, the relative likelihood of contributing to an opponent rises as the conditions more closely resemble the set of cases in the model: non-investor cases with no committee-industry match have the lowest propensity toward donation to opponents, and investor cases with a committee-industry match have the highest. Only the difference between the top and bottom category is significant at the .1 level. Figure 5 displays the results.

The theory does not predict a particular level of contributions to opponents: levels close to zero or close to one are all consistent with the model given different state distributions and parameter values. However, our interpretation of these data is that contributions to opponents are surprisingly high given many existing accounts of contribution behavior. Though the evidence is not confirmatory for our model it suggests that (a) contributing to opponents is a plausible strategy for donors to choose and (b) there is variation in the use of this strategy that could be the subject of future research that our model may help to guide. The respondent-level data reinforce both of these points. 

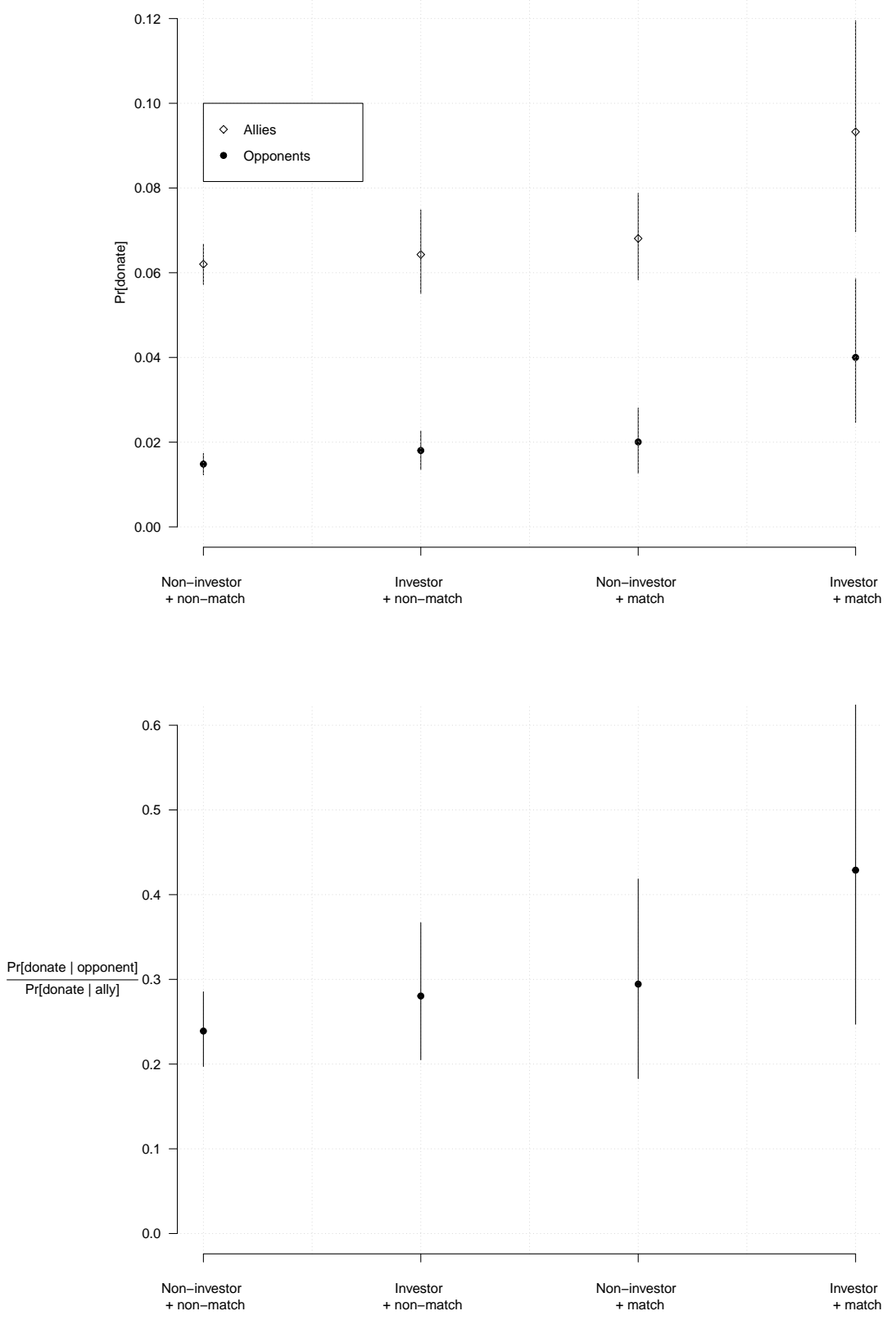

Figure 5: Top: The probability that a respondent donated to a particular Senator, conditional on ally/opponent status, committee match, and donor type. Bottom: The probability of donating to an opponent as a percentage of the probability of donating to an ally, conditional on committee match and donor type. Dots represent point estimates and line segments are $90 \%$ confidence intervals computed using a respondent-clustered bootstrap. 


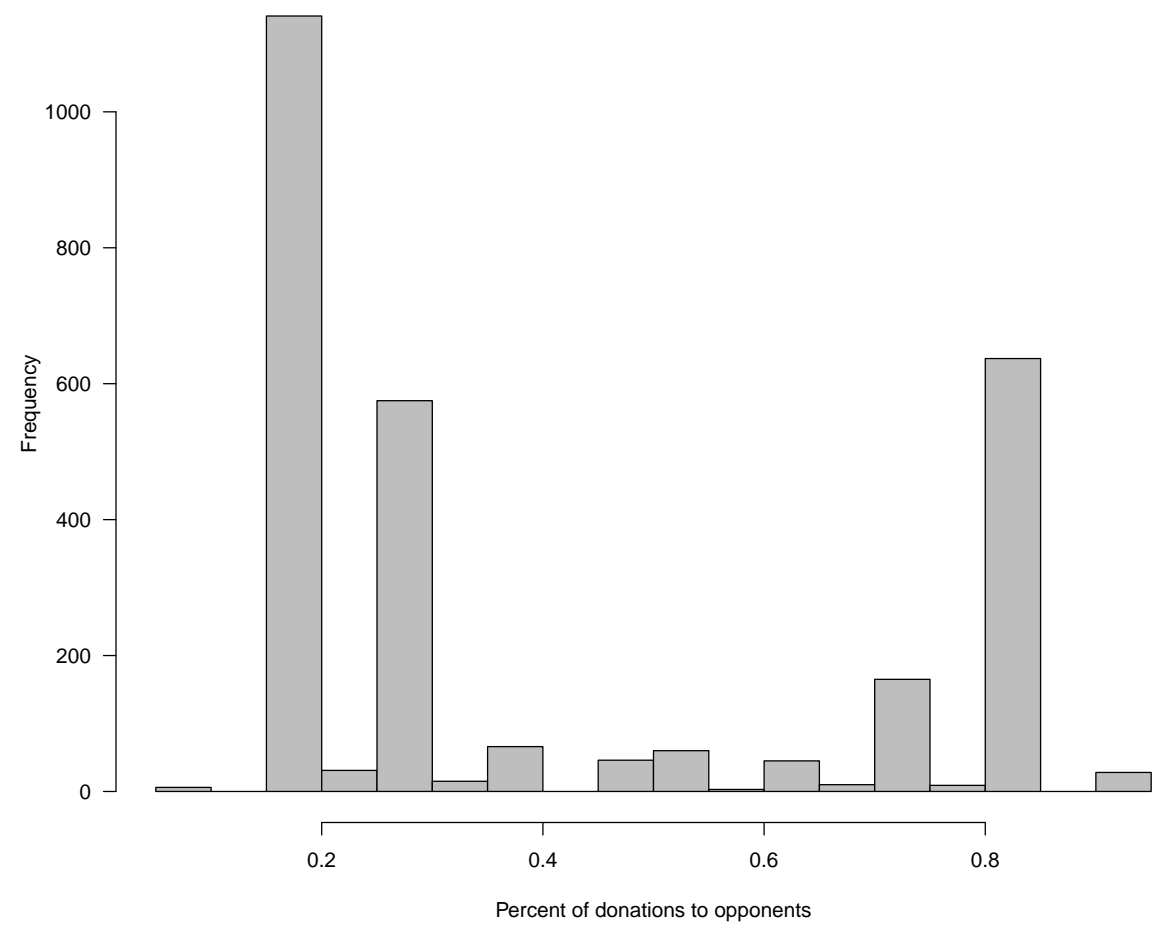

Figure 6: Histogram of the percentage of contributions going to opponents by donor

\section{C.3 Respondent-level statistics}

We now step away from the dyad-level analysis and aggregate the data by donor. This allows us to consider, for each donor, the percentage of contributions that go to opponents rather than allies. Our first observation is that the percentage of contributions to opponents across donors is bimodal and extremely variable. Figure 6 displays a histogram of this variable. As the figure shows, a sizable percentage of respondents gave substantially to opponents. For instance, around $23 \%$ of the respondents donated to opponents over $80 \%$ of the time when they contributed to campaigns. Figure 7 shows that some of this variation is explained by donor type: those that described themselves as donating for the sake of their business or industry were significantly more likely to give to opponents.

\section{C.4 Caveats}

We present this empirical analysis to demonstrate that contributing to opponents is a plausible contribution strategy for the donors of interest to us. We interpret the evidence to say that further research on the strategy of contributing to opponents may be fruitful and that this research may be usefully guided by our theoretical model. We are reticent to reach any stronger conclusions than that for several reasons. First, these data were not collected with our theoretical 


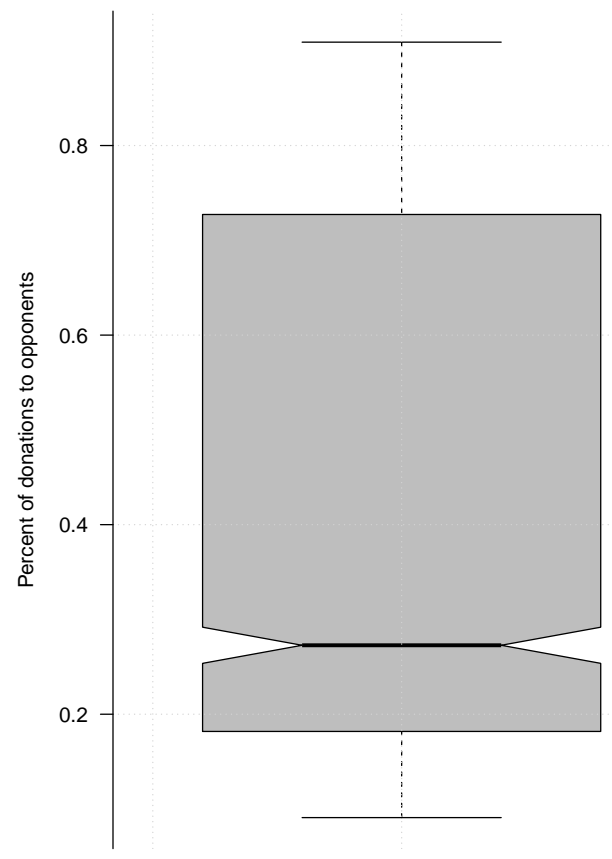

Non-Investor

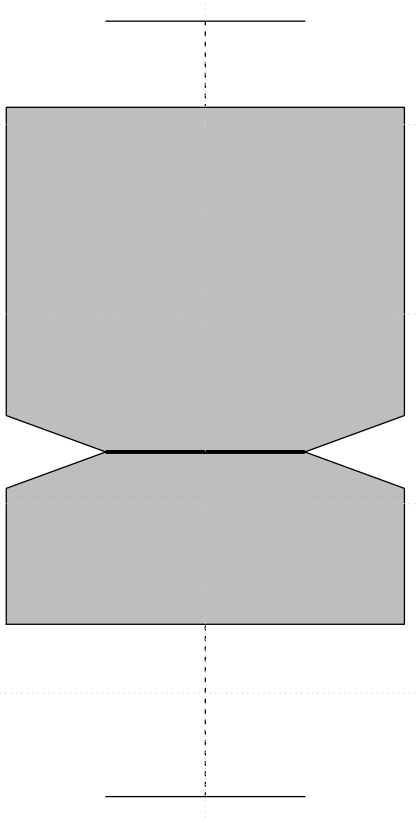

Investor

Figure 7: Box plot of the percentage of contributions going to opponents by donor, comparing donor types 
model in mind and the data probably cannot distinguish between our model and alternative explanations for this phenomenon. As we have described throughout the paper, the model has a number of empirical implications. However, a full empirical evaluation of the model is a larger undertaking which we think merits a separate research project.

Second, this dataset consists mostly of smaller donors. Though we have described how we think the model may extend to small donors, this was not the motivation of our study. Unfortunately, large donors also tend to contribute in ways that are harder for researchers to uncover. Our work-around for this in analyzing these data was to focus on self-described investor respondents in order to sort out most of those respondents giving for self-expression or consumption value. However, this categorization is far from perfect with respect to the attributes we are trying to highlight. Some ideological donors may indeed possess information about their pet issue and give in response to issue-specific information. Similarly, some investor donors may simply want to support candidates who are friendly to their industry and give in a more expression-oriented way. A serious attempt to confront the theory with data would likely need to collect original data and sample subjects in a way that emphasizes the characteristics of actors in our model. We would consider corporate and interest group PACs to be a better fit to the theory, though similar data for these groups was not available.

Third, though contributing to opponents is one of the more novel predictions from the model, and we wanted to demonstrate that such a strategy is plausible, it is not the best choice of outcome if we were attempting to subject the model to falsification. This is because the model is consistent with a variety of levels of donating to opponents, even in a separating equilibrium: we may have an equilibrium with persuasion by moderation which does not require actually contributing to the opponent or we may just have a small percentage of good types in the population. However, we consider this one piece of the puzzle that, along with the other empirical implications outlined in the paper, lay the groundwork for empirical applications of our model.

\section{References}

Barber, Michael, Brandice Canes-Wrone and Sharece Thrower. 2016. "Replication Data for: Ideologically Sophisticated Donors: Which Candidates Do Individual Contributors Finance?".

URL: https://doi.org/10.7910/DVN/DTFR60

Barber, Michael J., Brandice Canes-Wrone and Sharece Thrower. 2017. "Ideologically Sophisticated Donors: Which Candidates Do Individual Contributors Finance?” American Journal of Political Science 61(2):271-288.

Klumpp, Tilman, Hugo M. Mialon and Michael A. Williams. 2016. "The Business of American Democracy: Citizens United, Independent Spending, and Elections.” Journal of Law and Economics 59(1):1-43.

Milgrom, Paul R. 1981. "Good News and Bad News: Representation Theorems and Applications." The Bell Journal of Economics 12(2):380-391. 